\title{
The Sale of Multiple Assets with Private Information
}

\section{Zhiguo He}

Booth School of Business, University of Chicago

\begin{abstract}
By generalizing the Leland and Pyle (1977) model to the case of multiple correlated assets, this paper studies the signaling and hedging behavior of an intermediary who sells multiple assets in financial markets. Based on information asymmetry, this paper demonstrates the intrinsic interdependence of risk management and asset selling for intermediaries, and obtains several testable empirical implications. For instance, an intermediary with a more diversified underlying portfolio will face greater liquidity (a smaller price impact) when selling assets to the market. Several applications are discussed, including bank loan sales and selling mechanisms. (JEL D40, D82, G20)
\end{abstract}

Financial intermediaries manage and trade large portfolios of assets. For instance, Fannie Mae, a leading firm in the Mortgage Backed Securities (MBS) industry, issued 32 Fannie Mae MBS pools on 1 November 2004. ${ }^{1}$ Meanwhile, active risk management is becoming increasingly important for financial intermediaries, ${ }^{2}$ possibly due to the longterm capital management crisis in the fall of 1998.

Motivated by these facts, this paper generalizes the Leland and Pyle (1977, hereafter LP) model to study the multiasset trading behavior of financial intermediaries, which include banks engaging in loan sales and private equity funds selling shares of their ventures. According to information theory, intermediaries suffer from a lemon's problem, and have to convey the qualities of their assets through credible signals (e.g., the retention amount). However, multiple assets lead to a scenario where the intermediary seeks to minimize overall risk by selling positively correlated assets or holding negatively correlated ones. Such risk management behavior influences the signaling incentive

\footnotetext{
I thank my advisors in the Kellogg School of Management at Northwestern University, Mike Fishman and Arvind Krishnamurthy, for endless encouragement and support and the editor, Matthew Spiegel, for detailed instructions and insightful comments. I am also grateful to Bob McDonald, Alan Morrison (2006 AFA discussant), Vadim di Pietro, two anonymous referees, and participants at the AFA (2006) Boston meeting for numerous valuable suggestions. All errors are my own. Send correspondence to Zhiguo He, Booth School of Business, University of Chicago, 5807 South Woodlawn Ave., Chicago, IL 60637; telephone: 773-834-3769. E-mail: zhiguo.he@chicagogsb.edu.

1 Data source: Fannie Mae Web site, http://www.fanniemae.com/.

2 See, for instance, Allen and Santomero (1997), and others.
}

(C) The Author 2009. Published by Oxford University Press on behalf of The Society for Financial Studies. All rights reserved. For Permissions, please e-mail: journals.permissions@oxfordjournals.org. doi: $10.1093 / \mathrm{rfs} / \mathrm{hhn} 119$ 
for each asset, rendering the equilibrium pricing rules for all assets intrinsically interconnected.

Based on this "interconnectedness," this paper derives a two-dimensional equilibrium pricing system in closed form. In this cross-signaling equilibrium, the informed agent sends a two-dimensional vector signal - the selling fraction of each asset-to financial markets, and investors correctly price each asset by fully utilizing the two-dimensional signal. The notion of cross-signaling hinges on the interdependence of the agent's selling incentives for different assets. Under the LP framework, the agent signals an asset's quality by keeping a fraction of this asset; the greater the asset risk, the more credible the retention signal. Now, consider the case where the agent has two positively correlated assets. Holding more of asset 1 is not only a credible signal of a higher quality for asset 1 , but also a higher quality for asset 2 . The reason is that the extra retention of asset 1 increases the agent's risk exposure to asset 2 , and thus boosts her selling incentive for asset 2 . Consequently, if the agent maintains the same fraction of asset 2 in equilibrium, it must be the case that her marginal benefit of holding asset 2, i.e., its quality, is higher. The larger the correlation between assets, the higher the interdependence of selling incentives, and the greater the interconnectedness of equilibrium pricing rules. Similar logic holds for the negative correlation case; in fact, due to the explicit inside-hedging motive, this case clearly illustrates the interdependence between the endogenous risk management and signaling behaviors in a multi-asset framework.

The above intuition suggests that the intermediary's equilibrium hedging activity (holding of asset 2) plays a vital role when the intermediary is signaling her asset 1's quality through retention, and this model generates several novel predictions. For instance, all else equal, when assets are positively (negatively) correlated, holding more of asset 2 leads to a higher (lower) equilibrium price for asset 1 . And, the less correlated the assets, the smaller the assets' own-price impacts - that is, the negative price response to the asset's fraction sold will be lower. This result implies that an intermediary with a more diversified underlying portfolio faces a more liquid financial market (a smaller price impact) during the asset sale.

In Section 4, I discuss the model's application to bank loan markets. In the context of loan syndications, a recent empirical paper by Ivashina (2007) examines the impact of information asymmetry on the equilibrium loan pricing. Ivashina proposes a portfolio-based risk measure for individual loans that lead banks originate and place in the syndication market. In view of my theoretical model, her portfolio-based measure is much more appealing than the asset's individual risk, because as discussed above, the key determining factor for the asset's equilibrium price should be the asset's risk contribution relative to the lead bank's existing portfolio. However, the theoretical results in this paper call her identification strategy into question, and suggest that her estimate for the asymmetric-information cost is downward biased (therefore, a conservative estimate). 
Applying the model to the secondary loan market, this paper suggests that, holding selling fractions constant, banks with less geographically diversified lending bases will receive more favorable prices for their loan sales. Also, regarding the relation between the loan price impact and the covariance structure of concurrent loans sold by the same bank, my model suggests that the loan market will become more liquid (smaller price impacts) when loans on sale are mutually hedging (negatively correlated) assets. The model studied in this paper can also be applied to other financial intermediaries who engage in asset sales-for instance, private equity funds who sell their multiple ventures to financial markets within a short time window.

Section 5 considers several extensions. In Section 5.2, I compare different selling mechanisms available to intermediaries, where pooled sales and sequential sales are discussed. For uncorrelated assets, DeMarzo (2005) finds the "information destruction" effect of the pooled sale, because in the pooled sale the agent loses the flexibility to take different positions for each asset. By showing that separate sales dominate the pooled sale even for correlated assets, this paper generalized this "information destruction" effect. Also, early simultaneous (separate) sale dominates sequential sales, and the intermediary tends to accelerate the selling pace (selling asset 2 earlier with asset 1) given the additional concern of "cross-signaling." This provides another possible explanation for premature IPOs in the venture capital industry (e.g., Barry et al. 1990).

The rest of this paper is organized as follows. Section 1 provides a literature review. The model is presented in Section 2, and Section 3 derives separating equilibria for various cases. In Section 4, I discuss the model's application to bank loan sales. Section 5 considers extensions, and Section 6 concludes. All proofs are provided in the Appendix.

\section{Related Literature}

This paper is based on LP. In a simplified version of their model, there is a risk-averse agent with constant absolute risk aversion (CARA) utility $-e^{-r \widetilde{w}}$, where $\widetilde{w}$ is the agent's terminal wealth and $r$ is her risk aversion coefficient. The agent sells a fraction $\alpha \in[0,1]$ of her asset with payoff $\tilde{x}=\mu+\varepsilon$ to riskneutral investors, where $\varepsilon \sim \mathcal{N}\left(0, \sigma^{2}\right)$ ( $\mathcal{N}$ indicates the normal distribution) is the payoff innovation, and $\mu \in[\underline{\mu}, \infty)$ is the asset's quality. The quality $\mu$ is the agent's only private information, and in equilibrium investors correctly price the asset based on the agent's selling fraction.

Although the first-best outcome has the agent transfer her entire asset to investors at a fair price $\mu$, in a separating equilibrium, information asymmetry leads the market to form a downward-sloping pricing function $p_{L P}(\alpha)$. The Pareto-efficient equilibrium signaling schedule has the lowest-type agent sell her entire asset, i.e., $p_{L P}(1)=\underline{\mu}$. Given this, LP show that the equilibrium 
pricing function is

$$
p_{L P}(\alpha)=\underline{\mu}+r \sigma^{2}(\alpha-\ln \alpha-1) \quad \text { for } \alpha \in(0,1] .
$$

Note that $p_{L P}^{\prime}(\alpha)$ is negative, suggesting that financial markets become illiquid (a negative price impact) due to information asymmetry, and prices are lower for assets with larger selling fractions.

Relaxing the common-knowledge assumption about the asset variance $\sigma^{2}$, Hughes (1986) and Grinblatt and Hwang (1989) explore the two-dimensional private information issue. In comparison, by considering a multi-asset version of LP, this paper focuses on the two-dimensional private information pertaining to the assets' qualities, and keeps the assets' covariance matrix as common knowledge. More importantly, my model demarcates itself from Hughes (1986) and Grinblatt and Hwang (1989) in another key respect. In their models, the equilibrium variance schedule is the market's perceived asset's variance. However, the perceived variance does not enter the agent's mean-variance objective directly; instead, the agent's risk exposure is determined by the asset's true variance. Therefore, in Hughes (1986) and Grinblatt and Hwang (1989), the agent has no incentives to signal her variance type to the market, and the crosssignaling incentives are absent. In contrast, in this paper, both assets' pricing functions (signaling schedules) enter the agent's payoff, and the agent will cross-signal both assets' qualities through her retention fractions of each asset.

A class of multi-asset equilibrium pricing rules has been explored in the literature (e.g., Caballe and Krishnan 1994 and Bhattacharya, Reny, and Spiegel 1995 , etc.). These papers obtain a partially revealing equilibrium pricing system in a CARA-normality-noise setting. ${ }^{3}$ Bhattacharya, Reny, and Spiegel (1995) focus on the destructive inference among securities markets in an imperfectly competitive setting, and show that adding new assets may eliminate the trading of existing assets in equilibrium. In their model, the economy has a riskaverse informed agent, and a continuum of risk-averse, uninformed, but rational individuals. The informed agent receives random endowments that are unknown to the market; this "noisy endowment" serves a camouflage role similar to noise traders. In their model, the agent can take any unbounded position in each asset, as opposed to the selling fraction between $0 \%$ and $100 \%$ assumed in this paper. ${ }^{4}$ By an elegant convex analysis argument where the $D 1$ refinement and unbounded action space are utilized, the authors show that, when the size of the endowment noise is small relative to the extent of quality uncertainty, no equilibrium pricing system-linear or nonlinear — can exist with trading. In

3 Caballe and Krishnan (1994) have a risk-neutral agent, but with noise traders. In Bhattacharya, Reny, and Spiegel (1995), the informed agent receives noisy endowments. The theoretical advantage of partially revealing models, over the fully revealing one presented here, is their ability to obtain the equilibrium without certain prespecified boundary conditions. However, by excluding "noise," I am able to analyze the equilibrium pricing system and price impacts based only on the assets' payoff structure, without knowing the elusive characteristics of noise.

4 The assumption of a restricted action space of selling fractions is more realistic for certain businesses of financial intermediaries (e.g., bank loan sales and private equity funds). 
contrast, in the CARA-normality environment where their equilibrium pricing system with trading fails to exist, this paper derives a fully revealing equilibrium pricing system with trading, but without the aid of "noise."5

Besides the models of asset-selling motivated by risk sharing, DeMarzo (2005) considers a risk-neutral intermediary who sells assets because the intermediary has a higher discount rate. The higher discount rate is motivated by the idea that the intermediary can use the proceeds from the asset sales to purchase new undervalued assets. From a broader view, my paper also relates to the theory of multidimensional signaling, e.g., Engers (1987), and that of multidimensional screening, e.g., Chone and Rochet (1998); the latter authors focus on maximizing total profit rather than full separation.

\section{The Model}

\subsection{Setup}

Consider a risk-averse agent with CARA utility $-e^{-r \widetilde{w}}$, where $\widetilde{w}$ is the agent's terminal wealth, and $r$ is her risk aversion coefficient. In contrast to LP, suppose that the agent has two assets to sell, and at $t=0$ she simultaneously offers to sell fractions $\alpha \equiv\left(\alpha_{1}, \alpha_{2}\right)$ of each asset to risk-neutral investors. For example, the agent may be a bank engaging in loan sales, or a private equity fund selling shares of its ventures. Each selling fraction $\alpha_{i}$ belongs to the interval $[0,1]$, which precludes the agent from purchasing or short selling. Both restrictions naturally fit the practice of bank loan sales or private equity funds.

At $t=1$, the asset payoffs $\widetilde{\mathbf{x}}=\mu+\varepsilon$ are realized, where $\mu=\left(\mu_{1}, \mu_{2}\right)$ is the quality vector, and the innovation $\varepsilon=\left(\varepsilon_{1}, \varepsilon_{2}\right)$ is distributed as $\mathcal{N}(0, \Sigma)$, with the covariance matrix $\Sigma=\left[\sigma_{i j}\right]$. Denote $\sigma_{i}$ as the standard deviation of $\varepsilon_{i}$, and $\rho$ as their correlation. As in LP, $\mu_{i} \in\left[\mu_{i}, \infty\right)$ for $i=1,2$ is the agent's only private information. I refer to the agent type as the asset quality vector $\mu$.

As in LP, this paper derives a separating equilibrium. Faced by a twodimensional pricing system $\mathbf{p}(\alpha)=\left(p^{(1)}(\alpha), p^{(2)}(\alpha)\right)$, the agent $\mu$ optimally chooses $\alpha^{*}(\mu) \in \mathcal{A} \equiv[0,1]^{2}$ to maximize her mean-variance objective (recall the CARA utility and normal distribution):

$$
V(\mu, \alpha, \mathbf{p}(\alpha))=(\mathbf{1}-\alpha)^{\prime} \mu+\alpha^{\prime} \mathbf{p}(\alpha)-\frac{r}{2}(\mathbf{1}-\alpha)^{\prime} \Sigma(\mathbf{1}-\alpha) .
$$

In a separating equilibrium, since the market is risk-neutral and competitive, "market consistency" implies that these valuations are correct, i.e.,

$$
\mathbf{p}\left(\alpha^{*}(\mu)\right)=\mu .
$$

The agent sends her signals — selling fractions $\alpha$ - to investors, and the market utilizes these signals to correctly price both assets.

\footnotetext{
5 A "no trade equilibrium" always exists in both models.
} 
Denote the set of equilibrium strategies as $\mathcal{E} \subset \mathcal{A}$. The equilibrium strategy set $\mathcal{E}$ could be a strict subset of the action space $\mathcal{A}$, as certain selling strategies might be off-equilibrium. For simplicity, this paper searches for equilibrium pricing rules $\mathbf{p}(\cdot)$ that are smooth (continuously differentiable) on $\mathcal{E}$. Section 5.4 shows that the smoothness assumption is not key to the equilibrium properties derived in this paper.

\subsection{First-order conditions}

Fix any agent type $\mu$ and her optimal selling strategies $\alpha^{*}$. Maximizing (2) yields the first-order conditions (FOC) as

$$
\alpha_{1}^{*} p_{i}^{(1)}+\alpha_{2}^{*} p_{i}^{(2)}+r\left[\left(1-\alpha_{1}^{*}\right) \sigma_{i 1}+\left(1-\alpha_{2}^{*}\right) \sigma_{i 2}\right]=0 \quad \text { for } i=1,2, \quad(\mathrm{FOC})
$$

where $p^{(i)}\left(\alpha^{*}\right)$ is canceled with $\mu_{i}$ by market consistency (3), and denote the cross partial as $p_{i}^{(j)} \equiv \frac{\partial p^{(j)}}{\partial \alpha_{i}}$. Later I show that the value function $V(\mu, \alpha, \mathbf{p}(\alpha))$ is strictly concave in $\alpha$, and therefore Equation (FOC) is sufficient for optimality.

In Equation (FOC), the pricing-related term $\alpha_{1}^{*} p_{i}^{(1)}+\alpha_{2}^{*} p_{i}^{(2)}$ is the marginal benefit of retention due to price impacts, and $r\left[\left(1-\alpha_{1}^{*}\right) \sigma_{i 1}+\left(1-\alpha_{2}^{*}\right) \sigma_{i 2}\right]$ is the marginal cost of retention due to risk considerations. Because the covariance term (i.e., $\sigma_{12}$ or $\sigma_{21}$ ) contributes in the marginal cost for each asset, investors correctly ascertain that the selling incentives of these two assets are interconnected. Therefore, the price impacts must have nonzero cross-partials, i.e., $p_{i}^{(j)} \neq 0$. This is exactly the interesting cross-signaling effect-the retention of asset $i$ affects the pricing of asset $j$.

Applying the above argument for different $\mu$, with the (FOC) equations holding pointwise for each $\alpha$, we arrive at a system of partial differential equations (PDEs) for the equilibrium pricing system $\mathbf{p}(\cdot)$. Conveniently, this model exhibits an inherent symmetry, which reduces this PDE system to two separate linear PDEs. In the Appendix, I show that in equilibrium, the impact of asset 1's selling amount $\left(\alpha_{1}\right)$ on the price of asset $2\left(p^{(2)}\right)$ is the same as the price impact of $\alpha_{2}$ on $p^{(1)}$; that is,

$$
p_{1}^{(2)}(\alpha)=p_{2}^{(1)}(\alpha)
$$

Plugging this symmetry result back into Equation (FOC), one obtains a single PDE for asset $i$, where $p^{(j)}$ is no longer involved in the pricing of asset $i$ (omitting the superscript “ $*$ " on $\alpha$ ):

$$
\alpha_{1} p_{1}^{(i)}+\alpha_{2} p_{2}^{(i)}+r\left[\left(1-\alpha_{1}\right) \sigma_{i 1}+\left(1-\alpha_{2}\right) \sigma_{i 2}\right]=0 \quad \text { for } i=1,2 .
$$

Due to the cross-signaling effect, Equation (4) implies that asset 1's equilibrium price depends on not only its variance $\sigma_{11}$, but also its covariance $\sigma_{12}$ with asset 2. (A similar result holds for asset 2.) This raises the important distinction between the asset's individual variance and its portfolio-based risk contribution. In fact, the agent's asset 1 selling incentive-which is her marginal retention 
cost $\left(1-\alpha_{1}\right) \sigma_{11}+\left(1-\alpha_{2}\right) \sigma_{12}$ in Equation (4)—should be the incremental risk brought on by asset 1 given the agent's underlying portfolio. As a result, the portfolio-based risk measure $\left(1-\alpha_{1}\right) \sigma_{11}+\left(1-\alpha_{2}\right) \sigma_{12}$, rather than the risk measure based only on the asset's individual variance, is the key determining factor for the asset's equilibrium pricing rule. This portfolio-based risk measure can be estimated. For instance, Ivashina (2007) estimates each individual loan's risk contribution relative to the lead bank's existing portfolio, and uses this measure to identify the downward-sloping loan pricing schedule due to information asymmetry.

The equilibrium pricing system satisfies the PDE (4), which admits a closedform solution. The solution's exact form depends on the boundary conditions, which are the subject of the next subsection.

\subsection{Boundary conditions: The $\mathrm{BC}$ assumption}

To date, there are no solid theoretical foundations for boundary conditions in the multidimensional signaling literature. Similar to LP, in what constitutes the Riley outcome (Riley 1979), I characterize the Pareto-efficient separating equilibrium with the least amount of inefficient signaling.

In LP, the lowest-type agent sells her entire asset at a fair price. I preserve this feature by imposing analogous conditions on the agents with the lower-bound asset $\mu_{i}$. Let the first-best (without information asymmetry) selling strategy of asset $\bar{i}$, given $\alpha_{j}$, be

$$
\alpha_{i}^{F B}\left(\mu, \alpha_{j}\right) \equiv \underset{\alpha_{i} \in[0,1]}{\arg \max } V\left(\mu,\left(\alpha_{i}, \alpha_{j}\right), \mu\right)
$$

In words, $\alpha_{i}^{F B}$ is the agent's conditional first-best selling amount in asset $i$ where the conditioning is on the selling level of asset $j$. The quadratic form of $V$ in (2) implies that $\alpha_{i}^{F B}\left(\mu, \alpha_{j}\right)$ is independent of $\mu$; or, $\alpha_{i}^{F B}\left(\alpha_{j}\right)$ is a function of $\alpha_{j}$ only. For instance, when $\sigma_{12}>0$, we have $\alpha_{1}^{F B}\left(\alpha_{2}\right)=1$, since the agent will sell the entire asset 1 to minimize her risk exposure, regardless of her holding position of asset 2 .

To pin down the boundary pricing rules, this paper assumes that, in equilibrium, the agents with lower-bound asset $\mu_{i}$ sell the conditional first-best level $\alpha_{i}^{F B}\left(\alpha_{j}\right)$ of asset $i$. Put differently, the market identifies the agent that engages in asset $i$ 's conditional first-best selling strategy to be the type endowed with an asset $\mu_{i}$. This boundary condition generalizes the one in LP, and I call this the $\mathrm{BC}$ assumption. In reference to the previous example of $\sigma_{12}>0, \mathrm{BC}$ implies that, regardless of $\alpha_{2}$, if an agent sells her entire asset 1, then investors assign a value $\mu_{1}$ for this asset.

The resulting equilibrium is a natural generalization of the multidimensional Riley outcome, and inherits the Pareto efficiency property (given the trading restrictions imposed in this paper). Section 5.4 considers other Pareto-inefficient 
separating equilibria where $\mathrm{BC}$ is violated. ${ }^{6}$ Nevertheless, almost all key equilibrium properties continue to hold, suggesting that the results obtained in this paper are robust to the specific boundary conditions.

\section{Equilibrium Pricing System and Its Properties}

In this section, I first derive the equilibrium pricing system when the assets are positively correlated; then I turn to the negative correlation case. The general properties of these equilibrium pricing systems are discussed in Section 3.3.

\subsection{Positive correlation case}

Suppose that the assets are positively correlated, i.e., $\rho>0$. First, let us invoke $\mathrm{BC}$ to obtain the boundary pricing rules. Consider $\mu=\left(\mu_{1}, \mu_{2}\right)$; as discussed above, $\alpha_{1}^{F B}\left(\alpha_{2}\right)=1$. Intuitively, without informational problems, the agent with the lower-bound asset 1 should sell all of this asset, because holding asset 1 always worsens the agent's risk exposure by either its own variance, or the positive covariance with asset 2 . Hence, the BC assumption yields

$$
p^{(1)}\left(1, \alpha_{2}\right)=\underline{\mu_{1}} \quad \text { for } \forall \alpha_{2} \in[0,1] .
$$

Since the agent retains none of asset 1 , I am back to the LP single asset case for asset 2:

$$
p^{(2)}\left(1, \alpha_{2}\right)=\underline{\mu_{2}}+r \sigma_{22}\left(\alpha_{2}-\ln \alpha_{2}-1\right) \quad \text { for } \forall \alpha_{2} \in(0,1] .
$$

By symmetry, $p^{(2)}\left(\alpha_{1}, 1\right)=\mu_{2}$ for all $\alpha_{1} \in(0,1]$, and $p^{(1)}\left(\alpha_{1}, 1\right)=\mu_{1}+$ $r \sigma_{11}\left(\alpha_{1}-\ln \alpha_{1}-1\right)$ for all $\alpha_{1} \bar{\epsilon}(0,1]$.

In Figure 1, for any $\alpha^{0} \in \mathcal{A}$, the characteristic line, $L \equiv\left\{\alpha(t)=\alpha^{0} \cdot t: t \geq\right.$ $0\}$, is a ray that emanates from the origin $\mathbf{O}$, traverses $\alpha^{0}$, and then intersects with one of the boundaries $A_{i} \equiv\left\{\alpha \in \mathcal{A}: \alpha_{i}=1\right\}$ at $\alpha^{\prime}$. I have derived the pricing functions on both boundaries in the previous paragraph. Then, based on Equation (4) in Section 2.2, one can obtain $p^{(i)}\left(\alpha^{0}\right)$ by integrating along the ray from $\alpha^{0}$ toward $\alpha^{\prime}$, plus the boundary value at $\alpha^{\prime}{ }^{7}$

Let $\mathcal{A}_{i} \equiv\left\{\alpha \in \mathcal{A}: 0<\alpha_{j} \leq \alpha_{i}\right\}$; see Figure 1. For each individual asset $i$, the solution $p^{(i)}(\alpha)$ can be expressed as the sum of two components:

$$
p^{(i)}(\alpha)=p_{L P}^{(i)}\left(\alpha_{i}\right)+r \sigma_{12} \phi^{(i)}(\alpha),
$$

where $p_{L P}^{(i)}\left(\alpha_{i}\right)=\mu_{i}+r \sigma_{i i}\left(\alpha_{i}-\ln \alpha_{i}-1\right)$ is the LP single asset pricing function defined in Equation (1), with asset $i$ 's individual characteristics; and the

${ }^{6}$ In a unidimensional LP framework, this boundary condition can be justified by the belief consistency in sequential equilibrium (Mailath 1987). However, the multidimensional-type space makes the BC assumption in this paper stronger than this requirement. See Section 5.4 for details.

7 Along the characteristic line $L, \frac{d p^{(i)}(\alpha(t))}{d t}=\frac{1}{t} \sum_{j=1}^{2} \alpha_{j}(t) p_{j}^{(i)}=r \sum_{j=1}^{2}\left(\alpha_{j}^{0}-\frac{1}{t}\right) \sigma_{i j}$, and this term does not depend on $p^{(i)}$ itself. 


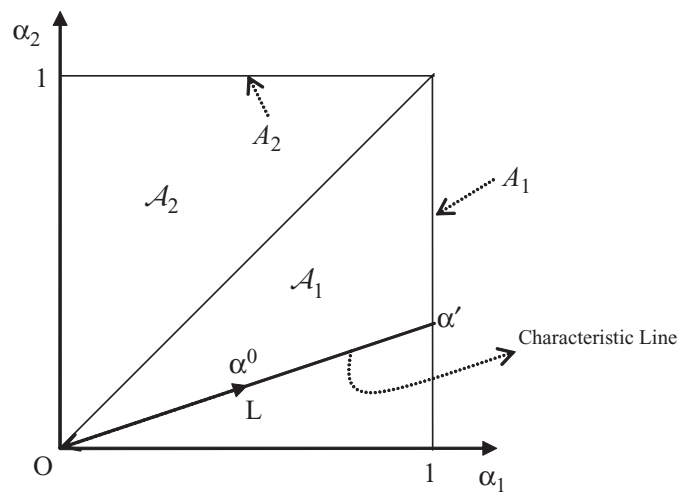

Figure 1

The positive correlation case

The characteristic line $L$ is a ray that emanates from the origin $\mathbf{O}$, traverses $\alpha^{0}$, and then intersects with one of the boundaries $A_{i} \equiv\left\{\alpha \in \mathcal{A}: \alpha_{i}=1\right\}$ at $\alpha^{\prime}$. The equilibrium strategy set is $\mathcal{E}=(0,1]^{2}$.

two-dimensional function $\phi^{(i)}(\alpha)$ is defined as

$$
\phi^{(i)}(\alpha)=\left\{\begin{array}{cl}
\alpha_{j}-\ln \alpha_{i}-\frac{\alpha_{j}}{\alpha_{i}} & \text { if } \alpha \in \mathcal{A}_{i} \\
\alpha_{j}-\ln \alpha_{j}-1 & \text { if } \alpha \in \mathcal{A}_{j}
\end{array},\right.
$$

where $j \neq i$ is the index for the other asset. A symmetric result holds for asset $j$.

One can show that $\phi^{(i)}(\alpha)$ is positive. ${ }^{8}$ Therefore, the equilibrium pricing function for asset $i, p^{(i)}(\alpha)$, is the LP unidimensional pricing function (1), plus an additional positive term, which corrects for the cross-signaling effect. When the assets are uncorrelated (i.e., $\left.\sigma_{12}=0\right), p^{(i)}(\alpha)$ reduces to the LP pricing function for each asset. The next proposition states that $\mathbf{p}$ is an equilibrium pricing system. ${ }^{9}$

Proposition 1. Under the pricing system $\mathbf{p}=\left(p^{(1)}, p^{(2)}\right)$ as defined in Equation (5), $V(\mu, \alpha, \mathbf{p}(\alpha))$ in Equation (2) is strictly concave in $\alpha$. Hence, $\mathbf{p}$ is an equilibrium pricing system. This equilibrium satisfies the intuitive criterion, and is Pareto-efficient relative to all (smooth) separating equilibria.

The equilibrium pricing system $\mathbf{p}$ in Equation (5) shows that when assets are correlated, one asset's pricing will depend on the transaction terms of the other asset—in other words, individual pricing rules are interconnected. Figure 2 plots the equilibrium pricing system $\mathbf{p}(\alpha)$ for different levels of $\alpha_{2}$. One observes a higher price for asset 1 when the agent retains more of asset 2 . Intuitively,

${ }^{8}$ For instance, when $\alpha \in \mathcal{A}_{1}$, then $0 \leq \alpha_{1}-\ln \alpha_{1}-1 \leq \alpha_{2}-\ln \alpha_{1}-\frac{\alpha_{2}}{\alpha_{1}} \leq \alpha_{2}-\ln \alpha_{2}-1$.

9 On the boundary $\left\{\alpha \in \mathcal{A}: \alpha_{i}=0\right\}, p^{(i)}$ diverges to $\infty$ (a similar result holds in the LP case). Therefore, strictly speaking, the equilibrium strategy set $\mathcal{E}=(0,1]^{2}$. I can simply set the off-equilibrium pricing rule as $\mathbf{p}=\underline{\mu}=\left(\underline{\mu_{1}}, \underline{\mu_{2}}\right)$. 

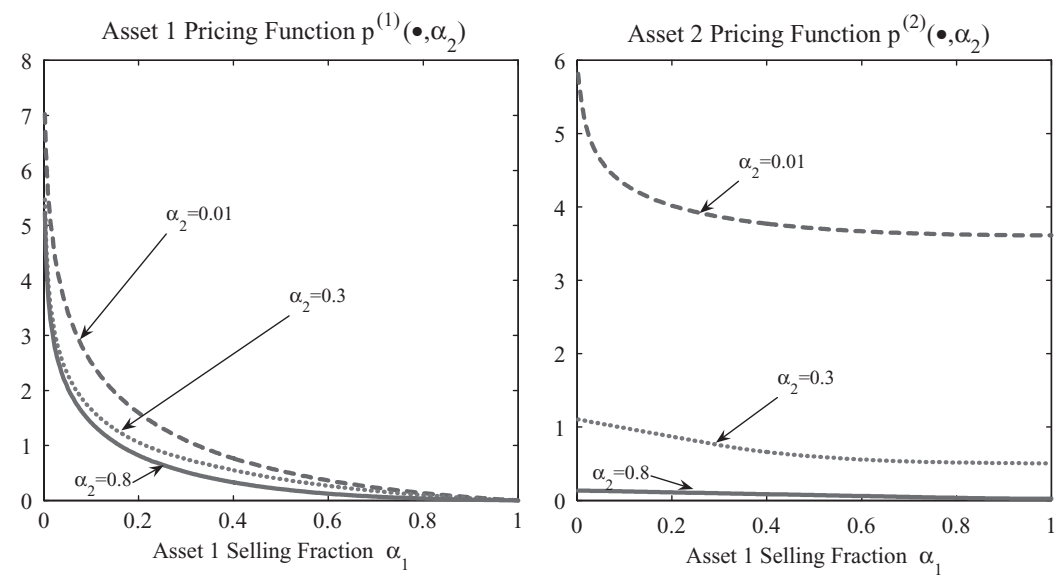

Figure 2

A numerical example of the equilibrium pricing system $\mathbf{p}(\alpha)$

In this example, $r \sigma_{11}=r \sigma_{22}=1, \rho=0.5$, and $\mu=(0,0)$. I plot $p^{(i)}$ 's against $\alpha_{1}$ when $\alpha_{2}$ takes value $0.01,0.3$, or 0.8. Due to symmetry, same results hold for $\bar{p}^{(i)}$ 's against $\alpha_{2}$.

when more of asset 2 with $\sigma_{12}>0$ lies in the underlying portfolio, holding asset 1 becomes more costly in terms of the portfolio-based risk exposure. This convinces the market that the marginal benefit of holding asset 1 -which is just the quality of asset 1 -is higher.

In addition, since the extra cross-signaling terms $\phi^{(i)}$ 's in Equation (5) are positive (see footnote 8 ), one also observes that each pricing function in $\mathbf{p}$ is higher than the LP pricing function in Equation (1). This implies that the higher the $\rho$, the stronger the cross-signaling effect, and therefore the higher the asset price. A testable implication is that, when institutions sell their assets, ceteris paribus, investors will attach a higher price to each individual asset when assets payoffs have a higher correlation. For instance, local banks who face less geographically diversified lending bases may be these institutions with highly correlated assets to sell. Empirical tests can also be conducted in the context of venture capital funds, as they usually specialize in particular industries (e.g., Sorenson and Stuart 2001) and sell fractions of their portfolio firms either via IPOs or to other private equity funds.

The relation between asset sale and asset correlation. The separating equilibrium in Proposition 1 has an interesting comparative static result, which holds in the negative correlation case as well. Fix the asset qualities $\mu_{1}$ and $\mu_{2}$, and write $\sigma_{12}$ as $\rho \sigma_{1} \sigma_{2}$ in Equation (5). The sign of $\frac{d \alpha_{i}^{*}}{d \rho}$, i.e., the effect of the correlation $\rho$ on the equilibrium selling fractions $\alpha^{*}$, is ambiguous. This counterintuitive - yet intriguing — result is unique to the two-dimensional equilibrium pricing system. The simple unidimensional LP intuition might suggest that $\frac{d \alpha_{i}^{*}}{d \rho}>0$, because the direct effect of a higher correlation leads to a greater 
risk exposure, which induces the agent to sell more of both assets in equilibrium. However, it turns out that the sign of $\frac{d \alpha_{i}^{*}}{d \rho}$ depends on the relative quality between these two assets. ${ }^{10}$ Intuitively, when asset 2 is much better than asset 1 , a higher $\rho$ necessarily leads to more selling of the low-quality asset 1 (a larger $\left.\alpha_{1}^{*}\right)$. For highly correlated assets, the reduction of the asset 1 position greatly lowers the marginal retention cost of the higher-quality asset 2 . As a result, the agent will hold more of asset 2 (a smaller $\alpha_{2}^{*}$ ) in equilibrium.

The same reasoning implies that the relationship between the assets' correlation $\rho$ and the total loan sale proceeds $\alpha_{1}^{*} \mu_{1}+\alpha_{2}^{*} \mu_{2}$ is also ambiguous. As explained above, the direct effect, which is often termed the diversification hypothesis in the literature on bank loan sales (e.g., Demsetz 2000), predicts that banks will engage in more loan sales when their portfolios are less diversified. However, the indirect effect shows that the above relation, under some circumstances, can be reversed. In a study of banks' loan sale activities, Pavel and Phillis (1987) find a positive relationship between the volume of loan sales and the loan portfolio concentration, suggesting that the direct effect dominates in their sample. ${ }^{11}$

With more detailed data, one could explore this issue further. In particular, with data on individual loan prices and selling fractions (e.g., the data in Gorton and Pennacchi 1995), and loan correlations (usually proxied by the geographic concentration of bank loans), one could test the following prediction: When a bank has highly correlated loans with divergent qualities (prices), one might expect the indirect effect to prevail (i.e., the loan sale volume becomes negatively related to the loan portfolio concentration).

\subsection{Negative correlation case}

3.2.1 Inside hedging needs. Now consider the case where the two assets are negatively correlated, $\rho<0$. From an empirical point of view, this is an equally important case - as risk management becomes increasingly important in today's intermediaries (e.g., Allen and Santomero 1997), intermediaries are prone to originate loans with negatively correlated payoffs.

When assets are negatively correlated, a complication arises due to the agent's inside hedging incentive: the retention of one asset, when $\rho<0$, can offset part of the risk associated with the other asset. This fact explicitly distinguishes this paper from LP, and underscores the underlying interdependence between endogenous hedging and signaling.

Let $\theta_{i} \equiv \frac{\sigma_{i j}}{\sigma_{i i}}=\frac{\sigma_{j} \rho}{\sigma_{i}}<0$. For the agent $\left(\mu_{1}, \mu_{2}\right)$ who is selling an $\alpha_{2}$ fraction of asset 2 in equilibrium, let us consider his conditional first-best selling strategy

10 Since $\frac{d \alpha^{*}}{d \rho}=-\left[\frac{\partial \mathbf{p}}{\partial \alpha}\right]^{-1}\left[\frac{\partial \mathbf{p}}{\partial \rho}\right]$, it can be verified that if $\alpha_{2}^{*} \ll \alpha_{1}^{*}$ and $\rho$ is large, then $\frac{d \alpha_{2}^{*}}{d \rho}<0$, i.e., the higher the $\rho$, the less the asset 2 sold by the agent.

11 Both Pavel and Phillis (1987) and Demsetz (2000) study a Logit model to explain the likelihood of a bank to engage in loan sales; and Pavel and Phillis (1987) also conduct a Tobit estimation where the loan sale volume becomes the dependent variable. The above discussion only applies to the study of Tobit estimation. 


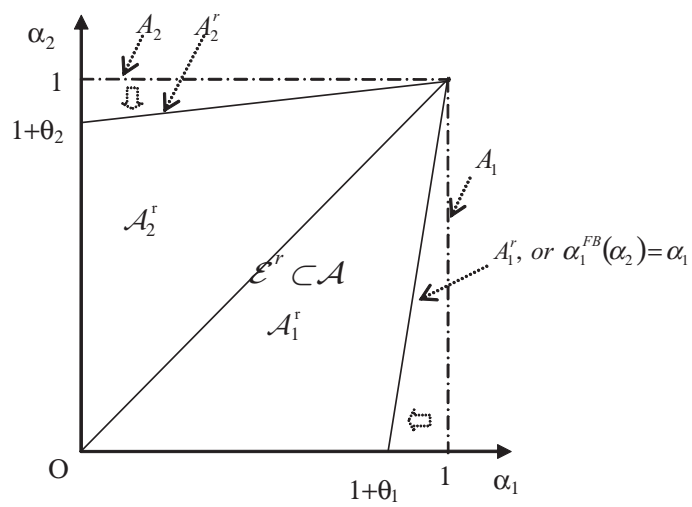

Figure 3

The regular case where assets are negatively correlated

The effective equilibrium strategy set $\mathcal{E}^{r}$ is the area between the lines $A_{1}^{r}=\left\{\alpha \in \mathcal{A}: \alpha_{1}=\alpha_{1}^{F B}\left(\alpha_{2}\right)\right\}$ and $A_{2}^{r}=$ $\left\{\alpha \in \mathcal{A}: \alpha_{2}=\alpha_{2}^{F B}\left(\alpha_{1}\right)\right\}$.

for asset 1 . Instead of $\alpha_{1}^{F B}\left(\alpha_{2}\right)=1$ for the positive $\rho$ case, when $\rho<0$, the internal hedging motive guides the agent to set (let $a \vee b \equiv \max (a, b))$ :

$$
\alpha_{1}^{F B}\left(\alpha_{2}\right)=0 \vee\left[1+\left(1-\alpha_{2}\right) \theta_{1}\right] \in[0,1] .
$$

In other words, given asset 2's retention fraction $1-\alpha_{2}$, the optimal hedging (holding) position for asset 1 is either $-\left(1-\alpha_{2}\right) \theta_{1}$, or binds at 1 (she keeps the entire asset 1 ). Define the boundary as

$$
A_{1}^{r} \equiv\left\{\alpha \in \mathcal{A}: \alpha_{1}=\alpha_{1}^{F B}\left(\alpha_{2}\right)\right\},
$$

anticipating that, in the spirit of $\mathrm{BC}$, in equilibrium the agent with $\mu_{1}$ will be on this boundary. Note that I simply rotate $A_{1}$ inside to $A_{1}^{r}$ due to the optimal hedging needs (see Figure 3). By the same token, define the boundary $A_{2}^{r} \equiv\left\{\alpha \in \mathcal{A}: \alpha_{2}=\alpha_{2}^{F B}\left(\alpha_{1}\right)\right\}$ on the other side, where $\alpha_{2}^{F B}\left(\alpha_{1}\right)=0 \vee[1+$ $\left.\left(1-\alpha_{1}\right) \theta_{2}\right]$.

This paper classifies the assets as regular if $A_{i}^{r} \in i n t \mathcal{A}_{i}$, or if $1+\theta_{i}>0$ for $i=1,2$, and irregular otherwise. If assets are regular, then $\left|\sigma_{12}\right|$ is dominated by $\sigma_{i i}$ for each asset, and $\alpha_{i}^{F B}$ 's will never bind at zero. As I will elaborate on in Section 5.1, the economic distinction between these two cases is that, in the regular case, the risk-shedding incentives in LP always dominate the inside hedging needs. ${ }^{12}$ Geometrically, in the regular case, two boundaries (i.e., $A_{i}^{r}$ 's) lie on different sides of the diagonal line (see Figure 3); whereas in the irregular case, both boundaries fall on the same side.

In the next subsection, I construct the equilibrium pricing system $\mathbf{p}^{r}$ for the regular case. The analysis for the irregular case is more involved; to focus on

12 In the irregular case, $\alpha_{i}^{F B}$ binding at zero implies that, without trading restrictions, the agent tends to purchaserather than sell, as in LP-asset $i$ for hedging purposes. See Section 5.1 for details. 
the central results and related empirical implications, I discuss the construction of the equilibrium pricing system for the irregular case in Section 5.1.

3.2.2 Regular case. Applying the same idea as in the $\rho>0$ case, the BC implies that $p^{r(1)}\left(\alpha_{1}^{F B}\left(\alpha_{2}\right), \alpha_{2}\right)=\underline{\mu_{1}}$ on $A_{1}^{r}$. A similar result holds for $p^{r(2)}$ on $A_{2}^{r}$.

Now I derive $p^{r(1)}$ on $A_{2}^{r}$ where sit the types with $\mu=\left(\mu_{1}, \mu_{2}\right)$. Due to the optimal hedging from asset 2 on $A_{2}^{r}$, the agent's total risk exposure is $\left(1-\alpha_{1}\right)^{2}\left(1-\rho^{2}\right) \sigma_{11}$, and she essentially faces a unidimensional LP problem with a variance $\left(1-\rho^{2}\right) \sigma_{11}$. Therefore, I have

$$
p^{r(1)}\left(\alpha_{1}, \alpha_{2}^{F B}\left(\alpha_{1}\right)\right)=\underline{\mu_{1}}+r\left(1-\rho^{2}\right) \sigma_{11}\left(\alpha_{1}-\ln \alpha_{1}-1\right) \quad \text { for } \forall \alpha_{1} \in(0,1] .
$$

Similarly, one can obtain $p^{r(2)}$ on $A_{1}^{r}$. Let $\mathcal{A}_{i}^{r} \equiv\left\{\alpha \in \mathcal{A}_{i}: \alpha_{i} \leq \alpha_{i}^{F B}\left(\alpha_{j}\right)\right\}$, and $\mathcal{E}^{r} \equiv \cup_{i=1}^{2} \mathcal{A}_{i}^{r}$ as the equilibrium strategy set for the regular case (see Figure 3). Similar to the positive correlation case, based on Equation (4) I derive $\mathbf{p}^{r}(\alpha)$ on $\mathcal{E}^{r}$, and each individual pricing function is

$$
p^{r(i)}(\alpha)=p_{L P}^{(i)}\left(\alpha_{i}\right)+r \sigma_{12} \phi^{r(i)}(\alpha),
$$

where $p_{L P}^{(i)}\left(\alpha_{i}\right)=\mu_{i}+r \sigma_{i i}\left(\alpha_{i}-\ln \alpha_{i}-1\right)$ is the LP single asset pricing function as defined in Equation (1), with asset $i$ 's individual characteristics; and the two-dimensional function $\phi^{r(i)}(\alpha)$ is defined as

$$
\phi^{r(i)}(\alpha)= \begin{cases}\frac{\sigma_{i i}}{\sigma_{12}} \ln \frac{\alpha_{i}\left(1+\theta_{i}\right)}{\alpha_{i}+\theta_{i} \alpha_{j}}+\alpha_{j}-\ln \frac{\alpha_{i}+\theta_{i} \alpha_{j}}{1+\theta_{i}}-1 & \text { if } \alpha \in \mathcal{A}_{i}^{r} \\ \frac{\sigma_{12}}{\sigma_{j j}} \ln \frac{\alpha_{i}\left(1+\theta_{j}\right)}{\alpha_{j}+\theta_{j} \alpha_{i}}+\alpha_{j}-\ln \frac{\alpha_{j}+\theta_{j} \alpha_{i}}{1+\theta_{j}}-1 & \text { if } \alpha \in \mathcal{A}_{j}^{r}\end{cases}
$$

where $j \neq i$ is the index for the other asset. A symmetric result holds for asset $j$.

By design, the area $\mathcal{A} \backslash \mathcal{E}^{r}$ consists of off-equilibrium strategies where the agent sells more of asset $i$ than the conditional first-best amount $\alpha_{i}^{F B}\left(\alpha_{j}\right)$. To deter these strategies, I simply set the harshest penalty-the lower-bound quality vector $\underline{\mu} \equiv\left(\underline{\mu_{1}}, \underline{\mu_{2}}\right)$-for these strategies. The following proposition holds.

Proposition 2. The pricing system $\mathbf{p}^{r}$ in Equation (6) delivers a separating equilibrium when two assets are regular. The equilibrium satisfies the intuitive criterion, and is Pareto-efficient relative to all (smooth) separating equilibria.

One can easily check that, when assets are uncorrelated, the pricing function in Equation (6) reduces to the unidimensional LP pricing function in Equation (1). And, relative to LP, Proposition 3 in Section 3.3 will show that, ceteris paribus, the prices in the equilibrium pricing system (6) are lower. ${ }^{13}$ The

13 Similar to the positive correlation case, $\phi^{r(i)}(\alpha) \geq 0$. But since $\sigma_{12}<0$, now the prices are lower. 
intuition is just the same as the positive $\rho$ case discussed in Section 3.1, but with an opposite force: Now the possibility of internal hedging dampens the agent's selling incentives, and the signal of asset retentions becomes less credible. Consequently, this leads to a lower price given the same selling fractions.

In addition to the price predictions discussed above, one can further verify that, when assets are more negatively correlated (a smaller $\rho$ ), each asset's ownprice impact $\left|\frac{\partial p^{r(i)}}{\partial \alpha_{i}}\right|$, which measures the information-driven illiquidity faced by the agent (as in LP and Kyle 1985), is smaller. Note that intermediaries with well-diversified underlying portfolios are endowed with assets that exhibit a smaller (more negative) $\rho$. For instance, large commercial banks tend to have geographically diversified lending bases, and skilled private equity funds with a broad investment focus will engage in advanced risk management in selecting their portfolio firms. Given this interpretation, the comparative static result regarding the relation between $\left|\frac{\partial p^{r(i)}}{\partial \alpha_{i}}\right|$ and $\rho$ suggests a negative relation between the "price impact" and the "portfolio diversification" for financial institutions. One immediate empirical prediction is that large commercial banks with crossstate lending bases should have smaller price impacts in their secondary loan markets activities.

Based on the equilibrium pricing system (6), I analyze the irregular case in Section 5.1. There, I show that the same pricing system applies, but with a different equilibrium strategy set.

\subsection{Properties of equilibrium pricing system}

Proposition 3 collects the general properties of equilibrium pricing systems.

Proposition 3. Let $\mathbf{p}$ be the equilibrium pricing system for all cases $(\rho>0$, regular, and irregular).

1. Cross-signaling effect. When $\rho>0(\rho<0)$, a larger $\alpha_{j}$ implies a lower (higher) price $p^{(i)}$ for asset $i$. And, the larger the correlation $\rho$, the higher the asset prices.

2. Price impact. Each equilibrium pricing function is downward-sloping in its own selling fraction, i.e., $p_{i}^{(i)}<0$. Moreover, the larger $\rho$, the more negative (or steeper) the own-price impact.

3. Equilibrium payoff. The agent's equilibrium payoff is increasing in $\mu$, and decreasing in $\rho$.

The cross-signaling effect constitutes one of the paper's major contributions; for a numerical example, see Figure 2. The intuition is rather straightforward if asset 2 sits in the agent's underlying portfolio exogenously, and the agent cannot sell asset 2 (so $\alpha_{2}=0$ always). This case corresponds to the LP singleasset case, but with the asset 1's virtual variance being $\sigma_{11}+\sigma_{12}$. Consider an exogenous increase of asset 2's position, which leads to a higher $\sigma_{12}$; then the LP result will imply a higher asset 1 price, because asset 1 's retention becomes 
more credible. This paper shows that such a positive relation between pricing and virtual variance should hold even when asset positions are endogenously determined in the simultaneous sales, thus it should be a solid theory to test empirically.

The second property connects the intermediaries' price impacts during asset sales to the intermediaries' diversification of their underlying portfolios (see related discussion at the end of Section 3.2.2). As Property 1 (cross-signaling effect) and Property 2 (price impact) show, in this model, both the "price impact" and the "price" are positively related to the assets' correlation. The underlying connection is the agent's selling incentives, which are positively related to the assets' correlation. On the one hand, for separation purposes, the equilibrium price impact has to reflect the agent's selling incentives; on the other hand, higher selling incentives make the retention signal more credible, and therefore leading to higher prices.

The third property is intuitive: since the agent has a mean-variance objective, higher qualities (a higher mean), or a better inside-hedging opportunity (a lower variance), leads to a higher equilibrium payoff for the agent.

\section{Bank Loan Markets}

\subsection{Background}

The bank loan market includes two broad categories: (1) the syndicated loan market where a loan originates in, and is placed with, a number of banks, and (2) the secondary market where a bank sells part of an existing loan to other institutions. The rapid development of bank loan markets has elicited extensive attention from researchers (see, e.g., Ivashina 2007 and Sufi 2007 on loan syndications, and Gorton and Pennacchi 1995 on the secondary bank loan market). ${ }^{14}$

Undoubtedly, bank loan markets suffer from the adverse selection (i.e., lemon's) problem studied in this paper. The following quote from Dahiya, Puri, and Saunders (2001), which appears in Bank Letter (19 June 1995), illustrates this point: "An original lender on a $\$ 150$ million Bradlees credit reportedly sold a $\$ 5$ million piece of the revolver in a hurry last week, ..., sending the message ... that the lenders most familiar with Bradlees are not comfortable with the company's situation."

This paper examines the bank's simultaneous loan sales on a portfolio basis. The assumption of simultaneous trading of multiple assets seems appropriate. For instance, the sample in Gorton and Pennacchi (1995) consists of 872 individual loan sales made by a single bank from 20 January 1987 to 01 September 1988. Also, according to the Dealscan dataset, JPMorgan Chase

14 For early studies on bank loan sales, see, for example, Pennacchi (1988), who argues that the optimal loan sale should balance the moral hazard problem with the relative advantage in funding cost. For early work on loan syndications, see, for example, Simons (1993). 
was the lead bank for 310 syndicated facilities within the second half of 2002 . Moreover, because bank loan transactions rarely involve short positions, the trading constraint $\alpha_{i} \in[0,1]$ imposed in this paper fits this application well.

Other assumptions in this model warrant further discussion. The assumption of risk-neutral buyers is standard in the loan sale literature (e.g., Gorton and Pennacchi 1995). This assumption can be justified on the ground that, compared to the seller bank, buyer institutions who either purchase small shares of loans for portfolio diversification purposes (e.g., Demsetz 2000), or issue securities backed by these assets right away to other diversified institutions (e.g., MBS markets), should be far less concerned about the asset's idiosyncratic shocks. For instance, Ivashina (2007) reports that, in her loan syndication samples, "Lead bank's average share is a substantial 27\% [...] Average participant share is $6 \%$, and participants are likelier to sell or securitize their risk."

Also, this paper assumes that the agent's (in the current context, the seller's or the lead banks') holding positions are publicly observable. ${ }^{15}$ Put differently, buyers in this market know the seller's or lead banks' selling incentives due to risk considerations. Though somewhat demanding for individual investors, this assumption is more reasonable for the sophisticated institutional participants in this market. Following Ivashina (2007), they can at least (approximately) construct the seller bank's existing loan portfolio from Dealscan. ${ }^{16}$

\subsection{Related empirical work}

The empirical literature on the bank loan market provides indirect supporting evidence for this paper. Consistent with the agent's (here the seller's or lead banks') underlying risk-diversification motive upon which this model is built, Pavel and Phillis (1987) find that loan concentration increases the likelihood of a bank's engagement in loan sales, and Demsetz (2000) shows that geographically expansive branch networks reduce the bank's secondary market activity. However, both papers study bank-level data only. In contrast, Gorton and Pennacchi (1995) and Ivashina (2007) focus on the pricing of individual loans, and find that a larger retaining share of the lead (or seller) bank indicates a higher loan quality in the transaction. ${ }^{17}$

In the context of loan syndications, Ivashina (2007) argues that the retention share of a lead bank has both diversification and asymmetric information effects on the equilibrium loan pricing. The diversification effect-without any informational concerns - simply captures the transfer from the corporate borrower

15 During loan sales or loan syndications, the seller's or lead bank's identity is publicly known to the buyer institutions, which implies that its selling activity is common knowledge.

16 Beyond the signaling framework, another piece of salient (and maybe extreme) evidence regarding "observable asset positions" is the sizeable "fire-sale" discount for those distressed banks who cannot maintain a healthy loan loss reserve. See "Sales in Distress," by R. England, http://www.bai.org/bankingstrategies/2002-may-jun/sales/.

17 In Gorton and Pennacchi (1995), the agency issue stems from the seller bank's moral hazard problem. However, its implication is the same as that of a signaling (adverse-selection) framework: a larger retention share-by inducing more effort-improves the ex post loan quality, and therefore the buyer banks are willing to pay more ex ante. 
to the lead bank. Under this effect, the lead bank with a larger retention share will demand a higher loan spread (a lower price) for risk compensation. The more interesting asymmetric information effect is reflected in the pricing schedule formed by the less-informed participant banks. The underlying mechanism could be either an adverse selection story where the lead bank signals the loan's quality through retention as in LP and this paper, or a moral hazard problem where the lead bank exerts ex post unobservable monitoring effort based on its holding fraction as in Gorton and Pennacchi (1995), or a mixture of both. Ivashina shows that, empirically, the asymmetric information effect causes the loan price (spread) to increase (decrease) with the lead bank's retention share, and she measures the asymmetric information cost as the slope (pricing impact) of this downward-sloping pricing schedule.

In the empirical framework of Ivashina (2007), the observed spread-retention pairs are equilibrium intersection points between the asymmetric information and diversification curves described above. Therefore, as in the framework of a simultaneous equations model, to trace down the asymmetric information effect, one needs to find factors that shift the diversification curve, but without shifting the asymmetric information curve. Ivashina argues that the individual loan's risk exposure specific to the lead bank's existing portfolio is such a factor that it only affects the diversification curve. To be discussed shortly, this claim is inconsistent with the theoretical results in this paper.

To obtain this portfolio-based risk exposure of individual assets, Ivashina (2007) measures the loan's risk contribution relative to the lead bank's existing portfolio, which is the marginal change of the lead bank's loss variance matrix due to holding this loan (see Ivashina 2007 for details). As discussed earlier in Section 2.2, this treatment is especially appealing in view of this paper: Note that more than determining the loan's individual variance, this portfolio-based risk measure directly determines the lead bank's retention incentives, and in turn the equilibrium pricing rules.

However, contrary to Ivashina's (2007) identification assumption, my theoretical model shows that the participant banks' pricing schedule - the asymmetric information curve-does depend on the loan's portfolio-based risk exposure measured above. The reason is simply that the lead bank's selling incentives alter with this measure; and once participant banks take this into account, the pricing schedule changes accordingly. Interestingly, according to my theoretical model, this fact causes the asymmetric information cost estimated in Ivashina (2007) to be downward biased, a fact that strengthens her empirical findings. ${ }^{18}$

18 Take the retention-spread plot used in Ivashina (2007) where retention is on the horizontal $x$-axis, and consider the situation where the loan is riskier (specific to the lead bank). The upward-sloping diversification curve shifts up (the lead bank needs more compensation from the corporate borrower, therefore a higher spread). Now, because the retention signal becomes more credible given a worse hedging position (see Figure 2 in this paper), the downward-sloping asymmetric information curve shifts down (note that I use spread instead of price here). Therefore, the new equilibrium point, relative to the one without shifting the asymmetric information curve as assumed in Ivashina (2007), has both a smaller retention fraction and a lower spread. This implies that the slope (which is negative, but consider its absolute value here) in Ivashina (2007) is downward-biased relative to the true 


\subsection{Future empirical work}

The direct application of this paper lies in the secondary bank loan market. The growing empirical literature on secondary loan sales (e.g., Gande and Saunders 2005), uses the Loan Pricing Corporation/Loan Syndications and Trading Association (LSTA/LPC) Mark-to-Market Pricing dataset, which contains daily bid-ask price quotes aggregated across dealers. This newly available dataset, combined with other detailed loan transaction and bank-level asset-holding data, potentially opens the door to investigating a variety of interesting empirical questions. For instance, it would be desirable to revisit Gorton and Pennacchi (1995), and identify the downward-sloping pricing schedule based on a larger and more representative sample. ${ }^{19}$

By studying loan pricing in a multi-asset framework, this paper suggests several testable implications related to the bank's underlying portfolio. For instance, using Ivashina's (2007) portfolio-based risk contribution measurement mentioned above, one can carry out a potentially interesting test: As suggested in Section 3.3, holding the selling fraction constant, the market will attach a higher price for the loan with a higher portfolio-based risk contribution (i.e., the loan price is higher when the bank's existing portfolio is less diversified). Notice that a story with noncompetitive buyers - combined with a bargaining mechanism - can potentially lead to an opposite prediction, as the seller bank will be left with a worse outside option in this scenario.

More importantly, this paper highlights the simultaneous multiple loan transactions in the secondary market, and Proposition 3 in Section 3.3 gives several empirical predictions regarding the relation between asset retention positions and their prices. For instance, when a holding bank sells two positively correlated assets, it would be interesting to identify the cross-signaling effect as illustrated in Figure 2-that is, the pricing of loan 1 should respond positively when the bank sells less of its second loan.

Future research can also investigate the price impact in the secondary bank loan market. My model predicts that the price impact of loan sales becomes larger either when the bank faces an underdiversified existing portfolio, or when the loans on transaction are more positively correlated. To be more specific, in explaining the loan prices, the coefficient on the interaction between the loan's selling share and its portfolio-based risk contribution should be positive.

\footnotetext{
slope for a given asymmetric information curve. Finally, note that a complete analysis needs a full-blown model, because the pricing schedule required by participant banks - under a signaling framework - should interact with the diversification curve. I leave this possibility for future research.

19 Gorton and Pennacchi (1995) only study a sample of 872 loan sales conducted by a single bank. Using Ivashina's (2007) terminology, relative to loan syndications, two facts about secondary loan sales can facilitate a cleaner empirical identification for "asymmetric information effect," which causes a downward sloping pricing schedule. First, the corporate borrower no longer plays any role in secondary loan sales, therefore the confounding "diversification effect" in Ivashina (2007) (which captures the transfer from the borrower to the lead bank in a loan syndication) is absent in this market. Second, in contrast to the loan origination where usually the corporate borrower only has a limited number of relationship lenders, in the secondary market, the seller bank faces a more competitive group of buyers, and the price should be relatively more "fair."
} 
If this is indeed the case, then these empirical findings might help deepen our understanding of the potentially time-varying liquidity in this market. ${ }^{20}$ The current literature on default risk (e.g., Das et al. 2006) documents not only that the firms' defaults are cross-sectionally correlated, but also that the default correlations are time-varying over business cycles or major economic events. ${ }^{21}$ Based on the techniques in Das et al. (2006), one can estimate the default correlations for loans in the secondary bank loan market. Then, an interesting follow-up question would be, how much of the time-series liquidity variability in the secondary bank loan market can be explained by the movement of loan correlations over time?

It is worth noting that one can raise similar empirical questions for the asset selling behavior of private equity funds who sell their portfolio firms in financial markets. In addition, the model studied in this paper can also be applied to financially troubled hedge funds who conduct forced sales in a short time window; one example would be the meltdown of hedge funds under Bear Stearns in 2007 in the early stage of the recent ongoing subprime-mortgage crisis.

\section{Generalizations and Extensions}

In this section, for completeness of the model, I first construct the equilibrium pricing system of the irregular case. I then compare different selling mechanisms available to intermediaries, and move on to discuss the model's extension to general $n$-asset cases. Finally, I show that the resulting equilibrium properties obtained in Section 3.3 are robust to the specific boundary conditions (BC) imposed in this paper.

\subsection{Irregular case}

Before solving for the equilibrium pricing system of the irregular case, let us discuss the key economic distinction between the regular case and the irregular case. In the regular case, for any asset, the agent's inside hedging incentive is smaller relative to the outside risk-sharing motive. To see this, imagine that the agent decides to hold $\epsilon$ fraction more of asset 1 . Then her optimal (marginal) internal hedging demand for asset 2 is $-\theta_{2} \epsilon$. If $-\theta_{2}<1$, the agent still has $\left(1+\theta_{2}\right) \epsilon>0$ fraction of asset 2 left in her hands, and tries to sell it to the market for better risk-sharing. However, if $-\theta_{2}>1$, the agent actually wants to hold back more asset 2 than $\epsilon$ for optimal internal hedging. Therefore, for asset 2, marginally the agent wants to retain more, rather than sell more, even

${ }^{20}$ For data availability issue, there are few academic studies on this topic, even though some anecdotal evidence suggests so (e.g., "Sales in Distress," http://www.bai.org/bankingstrategies/2002-may-jun/sales/). Nevertheless, because the time-varying liquidity in the equity market is well documented, and because the secondary bank loan market requires more specialty, one would expect similar results as in the equity market one would expect at least similar results for the secondary bank loan market, if not stronger.

21 For a given period, the default correlation can come from either the correlation between default intensities as a standard doubly stochastic process predicts, or clustered actual defaults due to, say, contagion effects. See Das et al. (2007) for more details. 


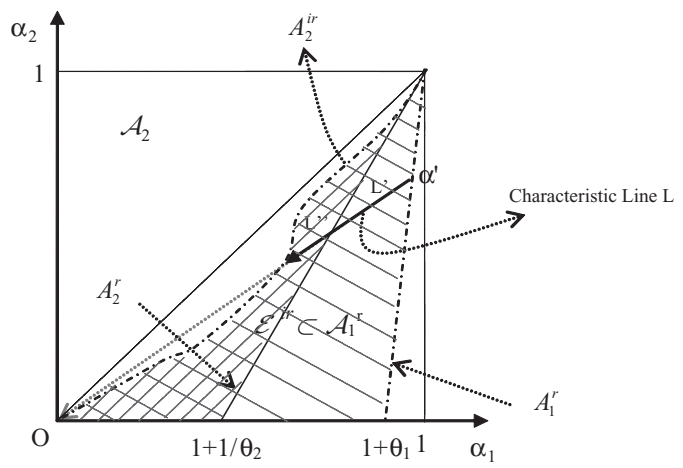

Figure 4

The irregular case where $\theta_{2}<-1$

The equilibrium strategy set $\mathcal{E}^{i r}$ is the total shaded area (including the simple-shaded area and the cross-shaded area) between $A_{1}^{r}$ and $A_{2}^{i r}$. In equilibrium, agents in the cross-shaded area (between $A_{2}^{r}$ and $A_{2}^{i r}$ ) sell more asset 2 than their (conditional) first-best hedging amount.

at a fair price $\mu_{2}$. In this sense, when $-\theta_{2}>1$, for asset 2 the agent's inside hedging incentive dominates her outside risk-sharing motive.

Based on the results in the regular case derived in Section 3.2.2, one can construct the equilibrium pricing system $\mathbf{p}^{i r}$ for the irregular case. Without loss of generality, suppose that $1+\theta_{2}<0$, or $-\sigma_{12}>\sigma_{22}$. Geometrically, the boundary $A_{2}^{r}$ is also located in $\mathcal{A}_{1}$ (see Figure 4). Using Equation (4), one can show that the asset 2 equilibrium pricing function $p^{i r(2)}$ achieves its maximum on $A_{2}^{r}$ along any characteristic line $L$ that intersects with $A_{2}^{r}$ (see Lemma 1's proof in the Appendix); therefore the BC assumption for asset 2 cannot hold. As discussed above, this result is rooted in the fact that, in the irregular case, for asset 2 the agent's outside risk-sharing motive is dominated by the inside hedging one, and therefore the standard LP intuition reverses. ${ }^{22}$

However, by keeping the BC for asset 1 only, the pricing system $\mathbf{p}^{r}$ defined in Equation (6) still delivers a separating equilibrium for the irregular case. Take $\mathbf{p}^{r}$ in Equation (6), and focus on $\mathcal{A}_{1}^{r}$; there exists a curve $A_{2}^{i r}$, which is located between the diagonal line $\left\{\alpha_{1}=\alpha_{2}\right\}$ and $A_{1}^{r}$ (see Figure 4), such that $p^{r(2)}(\alpha)=\mu_{2}$ for $\alpha \in A_{2}^{i r}$ (see the Appendix for details). Simply put, on the curve $A_{2}^{i r}, \overline{\mathbf{p}^{r}}$ prices asset 2 at its lower bound; or, agents with $\mu_{2}$ optimally choose selling strategies on $A_{2}^{i r}$. This suggests that the equilibrium strategy set $\mathcal{E}^{i r} \subset \mathcal{A}_{1}^{r}$ is the area between $A_{1}^{r}$ and $A_{2}^{i r}$ (the total shaded area-including both the simple-shaded area and the cross-shaded area-in Figure 4). The next proposition follows.

22 Recall that $\mathrm{BC}$ requires that types with $\mu_{2}$ (the lower bound) lie on $A_{2}^{r}$, contradicting the maximum of $p^{i r(2)}$ on $A_{2}^{r}$. The reason for this result is as follows. In the irregular case, because of the dominating inside-hedging benefit, the marginal retention cost $r\left[\left(1-\alpha_{1}\right) \sigma_{12}+\left(1-\alpha_{2}\right) \sigma_{22}\right]$ in Equation (4), which is positive in both the positive $\rho$ and regular cases (and also in the LP unidimensional case), turns negative, as $\sigma_{12}<0$ dominates $\sigma_{22}$. As a result, retention actually incurs some marginal benefit in the irregular case, which necessarily reverses the pricing effect. 
Proposition 4. Let $\mathbf{p}^{i r}(\alpha)=\mathbf{p}^{r}(\alpha)$ for $\alpha \in \mathcal{E}^{i r}$, and $\mathbf{p}^{i r}(\alpha)=\mu$ for offequilibrium strategies $\alpha \notin \mathcal{E}^{i r}$. Then, $\mathbf{p}^{\text {ir }}$ delivers a separating equilibrium for the irregular case. This equilibrium satisfies the intuitive criterion, and is Pareto-efficient relative to all (smooth) separating equilibria.

Although $\mathbf{p}^{i r}(\cdot)$ for the irregular case is the same as $\mathbf{p}^{r}(\cdot)$ for equilibrium strategies, they possess some distinct properties. Note that, along $A_{2}^{i r}$, the agent with $\mu_{2}$ has $\alpha_{2}^{*}>\alpha_{2}^{F B}\left(\alpha_{1}\right)$, as $A_{2}^{i r}$ is above $A_{2}^{r}$. In other words, agents who lie between $A_{2}^{i r}$ and $A_{2}^{r}$ - the cross-shaded area in Figure 4- "optimally" sell more of asset 2 than the conditional first-best hedging level. Therefore, for an agent lying on the cross-shaded area, retaining more of asset 2 incurs less risk exposure, as opposed to the original LP scenario where more retention always gives rise to more risk exposure. However, as shown in Proposition 3 in Section 3.3, similar to LP, the own-price impact of asset 2 is negative, i.e., $\frac{\partial p^{i r(2)}}{\partial \alpha_{2}}<0$. Therefore, this agent also receives a direct-signaling reward (a higher price) from the asset 2's extra retention. Then the intriguing question is, what drives the agent to sell more of asset 2 than her conditional first-best level $\alpha_{2}^{F^{B}}\left(\alpha_{1}\right)$ in equilibrium?

The answer is to cross-signal her asset 1's quality. Recall that, since choosing a higher $\alpha_{2}>\alpha_{2}^{F B}\left(\alpha_{1}\right)$ leads to a worse hedging position for asset 1, selling more of asset 2 is a credible signal for a higher quality of her asset 1 . In fact, in this case, this cross-signaling effect could be sufficiently strong to dominate the direct-signaling effect. To be precise, the following relation holds for the area close to $A_{2}^{i r}$ in Figure 4,

$$
\frac{\partial p^{i r(1)}}{\partial \alpha_{2}}>\left|\frac{\partial p^{i r(2)}}{\partial \alpha_{2}}\right|>0,
$$

which states that the asset 2 selling position has a stronger impact on asset 1 's pricing (cross-signaling) than does that of asset 2 (direct-signaling). ${ }^{23}$ This implies that, in some circumstances, cross-signaling (as opposed to the directsignaling) could be the leading concern during asset sales when the agent possesses multidimensional private information.

\subsection{Selling mechanisms}

5.2.1 Pooled sale. In this model, aside from selling separately, the agent has another option: packaging both assets and selling them as a single portfolio. Which selling strategy is optimal? DeMarzo (2005) finds that a separate sale dominates pooling when $\rho=0$, and he labels this the "information destruction"

23 This inequality (7) can be easily verified in a numerical example. The intuition of a dominant cross-signaling effect is as follows: in the irregular case, the covariance $\left|\sigma_{12}\right|$ dominates the individual variance $\sigma_{22}$ (or, $\left|\theta_{2}\right|>1$ ), and therefore the cross-signaling effect may prevail. In contrast, this result never holds in the regular case. 
effect of pooling. ${ }^{24}$ In this section, I show that this property remains true even if $\rho \neq 0$. Therefore, information destruction by pooling is significantly more general than what has been acknowledged in the current literature.

In a pooled sale, the agent sells to the market a fraction of the portfolio consisting of both assets. In other words, this is the LP single-asset case where the asset is the portfolio of both assets. The equilibrium selling fraction $\alpha$ is characterized by the LP single-asset pricing function (1) with $\underline{\mu}=\underline{\mu_{1}}+\underline{\mu_{2}}$, $\sigma^{2}=\sigma_{11}+2 \sigma_{12}+\sigma_{22}$, and $p_{L P}(\alpha)=\mu_{1}+\mu_{2}$. The agent's equilibrium payoff is

$$
\mu_{1}+\mu_{2}-\frac{r}{2}\left[(1-\alpha)^{2}\left(\sigma_{11}+2 \sigma_{12}+\sigma_{22}\right)\right]
$$

To verify the "information destruction" effect, it amounts to showing that the agent will face a larger risk exposure by pooling her assets before the sale.

Proposition 5. Separate sales dominate a pooled sale. The two mechanisms are equivalent if and only if $\frac{\mu_{1}-\underline{\mu_{1}}}{\sigma_{11}+\sigma_{12}}=\frac{\mu_{2}-\underline{\mu_{2}}}{\sigma_{22}+\sigma_{12}}$ in the positive correlation and regular case.

The above result is somewhat surprising since, for a risk-averse agent, it appears that pooling negatively correlated assets together has a "riskdiversification" benefit, a term used in DeMarzo (2005). However, the discussion in Section 3.2.1 suggests that the risk-diversification benefit from negatively correlated assets is present even without pooling. By pooling assets before the sale, the agent just loses the flexibility to take different positions on each asset. The principal implication is that, in DeMarzo (2005), the "riskdiversification effect," which favors pooling assets (and then tranching) is fundamentally different from the diversification benefit in the standard portfolio theory, where a risk-averse preference is assumed. Rather, the diversification effect in DeMarzo (2005) is similar to that in Diamond (1984) where the bank takes all individual loans and issues deposits to outside investors. In both papers, debt is the optimal contract, and the per-asset likelihood of a tail-event shrinks as the number of assets increases.

Of course, pooling assets may prove optimal for other reasons. Suppose that, before shedding the assets, the agent could exert certain unobservable monitoring effort to improve asset qualities. By the envelope theorem, the impact of asset $i$ 's quality on the agent's equilibrium payoff is simply $\frac{d V^{*}}{d \mu_{i}}=$ $1-\alpha_{i}$. Since the asset retention $\left(1-\alpha_{i}\right)$ is small when asset quality is low, the agent has little incentive to monitor low-quality assets, even though it might be socially optimal to exert a significant amount of effort on them. For instance,

24 The information destruction effect of pooling under the multi-asset LP case with $\rho=0$ is presented in the Appendix of DeMarzo (2003). In its final version (DeMarzo 2005), this analysis has been taken out. 
in the scenario of bank loans, equity holders of the borrower firm might gain substantially from the bank's effective monitoring.

5.2.2 Sequential sales. Another possible selling mechanism is sequential sales. For illustrative purposes, I discuss the case with a positive correlation; this case resembles a private equity specialty fund (or a bank) who sells multiple portfolio firms (or loans) within the same industry.

He (2005) considers the scenario where the payoff of the first asset $\left(\tilde{x}_{1}\right)$ is realized before the sale of the second asset (otherwise it is equivalent to a simultaneous sale), and derives a separating equilibrium in closed form. Investors will update asset 2's quality given asset 1's innovation, due to the positive correlation. Note that a separating equilibrium requires the agent's commitment on her selling fraction of the second sale at the time of asset 1 sale; otherwise, when selling asset 1 , the unidimensional signal cannot separate multidimensional types.

Interestingly, when comparing the agent's expected payoff from sequential sales with the expected payoff obtained from simultaneous sales before the realization of $\tilde{x}_{1}$, He (2005) finds that the simultaneous sale dominates the sequential sale. The reason is that the agent will be exposed to more risk when the market updates asset 2's quality due to new information about $\varepsilon_{1}$. Therefore the agent tends to accelerate her selling pace-i.e., selling her asset 2 earlier with asset 1 at the same time-given the additional concern of cross-signaling, and this finding provides another possible explanation for premature IPOs in the venture capital industry (e.g., Barry et al. 1990).

\subsection{General $\boldsymbol{n}$-asset cases}

By induction, one can construct the Pareto-efficient separating equilibrium for the general $n$-asset case. However, the tractability of construction crucially depends on the structure of the covariance matrix $\Sigma$.

When all assets are positively correlated, i.e., $\sigma_{i j} \geq 0$ for all $i$ and $j$, the equilibrium pricing system $\mathbf{p}$ is available in closed form. The tractability in this case stems from the simple equilibrium strategies for the boundary agents: when there is no internal hedging motive for any asset, BC implies that the agents with asset $\mu_{i}$ will set $\alpha_{i}=1$ and retain none of this asset. This reduces an $n$-asset problem to an $(n-1)$-asset problem, and allows me to derive a closedform $n$-dimensional equilibrium pricing system by induction in the Appendix. Though a bit more cumbersome, this system shares the same properties with the two-dimensional model (the pricing system in Equation (5)) derived in Section 3.1.

When assets have an arbitrary covariance matrix, internal hedging motives across different assets - coupled with trading restrictions - induce complicated equilibrium strategies for boundary agents. As a result, the boundary pricing rules are quite involved. In a 3-asset example studied in the Appendix, asset 1 and asset 3 are independent; however, asset 2 is positively (negatively) 
correlated with asset 1 (asset 3). Here, asset 2 serves two opposite roles in "hedging" the other two assets. In equilibrium, the agents endowed with $\mu_{2}$ sit on a kinked surface in $[0,1]^{3}$, reflecting the fact that the asset 2's first-best hedging strategy can be nonsmooth due to the complicated covariance structure and trading restrictions. This renders the nontractability of the general covariance matrix case.

Nevertheless, by an induction procedure, there are no conceptual difficulties in deriving the Pareto-efficient separating equilibrium for the higherdimensional case, and the key economic insights delivered in the 2-asset case will continue to hold. Similar to the derivation in Section 3.1, the induction step involves constructing the $m$-dimensional pricing systems (where $m=0,1, \ldots, n)$ for boundary types that possess $n-m$ assets with the lowerbound qualities (note that there are $\frac{n !}{m !(n-m) !}$ pricing systems to be solved for). Here, the $\mathrm{BC}$ assumption, which ensures that there is no private information in deriving $\mathbf{p}$ for these boundary types, is pivotal. Clearly, $m=0$ is the degenerate case (the pricing system is simply $\mu$ for the first-best selling strategies). Given the collection of pricing systems for $m \geq 0$, which might be complicated due to the optimal hedging positions from those $n-m$ boundary assets (see the previous 3-asset example), the construction of pricing systems for $m+1$ is merely a line integration in some appropriate $(m+1)$-dimensional space in $(0,1]^{n}$, based on the PDE similar to Equation (4), which involves $m+1$ unknown pricing functions. In the Appendix, interested readers can find the construction details for the equilibrium pricing system for the 3-asset case mentioned above.

\subsection{Boundary conditions and Pareto-inefficient separating equilibria}

As discussed in Section 2.3, the boundary condition assumption BC is designed to pick out the Pareto-efficient Riley outcome. Unlike the unidimensional LP case (see footnote 6), under the current multidimensional signaling framework, one can construct a continuum of Pareto-inefficient separating equilibria that satisfy both the belief consistency requirement and the intuitive criterion. The Appendix gives such an example with $\rho>0$; in this inefficient equilibrium, the agents endowed with $\mu_{1}$ optimally choose $\alpha_{1}<1$ (as opposed to $\alpha_{1}^{F B}=1$ in the equilibrium derived in Section 3.1), because selling the entire asset 1 incurs a substantial penalty through the asset 2 pricing rule. However, almost all key properties established in Proposition 3 continue to hold in these equilibria.

In this inefficient equilibrium, the individual pricing functions are nonsmooth (i.e., not continuously differentiable on the equilibrium strategy set $\mathcal{E}) .{ }^{25}$ Therefore, the "smoothness" assumption about individual pricing functions is not crucial for the key equilibrium properties derived in this paper.

Admittedly, these Pareto-inefficient examples are far from constituting solid theoretical grounds for equilibrium refinement in the multidimensional

25 However, the agent's payoff function $V$, as a composite function of the entire system $\mathbf{p}$, continues to behave smoothly. 
signaling game. However, they advance our understanding of the multiplicity of separating equilibria. In addition, the results here demonstrate that the key properties of the Pareto-efficient equilibrium are robust within a wide class of equilibria.

\section{Concluding Remarks}

This paper studies the signaling and hedging behavior of financial intermediaries in a multi-asset environment. By generalizing LP's result, this paper develops a multidimensional equilibrium pricing system for correlated assets, and offers a framework to analyze the interdependence between asset selling and risk management for financial intermediaries. By acknowledging the interconnectedness between any asset and the portfolio of the informed owner, I derive the cross-signaling patterns that arise in equilibrium. In short, the agent's holding position of asset $i$ reveals information about her asset $j$ 's quality.

The resulting equilibrium pricing system allows one to examine the crosssectional pattern in asset prices and the price impacts faced by financial firms, and provides several interesting testable empirical predictions. For instance, in the presence of information asymmetry, financial firms with underdiversified portfolios should have larger price impacts, and therefore suffer greater illiquidity problems when selling their assets. This prediction is robust to a wide range of equilibria, as well as the possibility of active hedging performed by the agent (see He 2005).

This paper solves a linear PDE system in closed form. This novel technique can be potentially applied to other CARA-normality models in finance. Similar to LP, I derive the Pareto-efficient separating equilibrium (a multidimensional version of Riley outcome), and discuss the empirical predictions based on the resulting equilibrium pricing system where the individual pricing functions are interconnected. I also construct a continuum of Pareto-inefficient separating equilibria that satisfy both intuitive criterion and belief consistency. Hopefully, these results can shed some light on general theories about multidimensional signaling games in future work, especially on the theoretical grounds for boundary conditions.

\section{A. Appendix}

Throughout the Appendix, I denote $\Xi \equiv\left[\mu_{1}, \infty\right) \times\left[\mu_{2}, \infty\right)$ as the agent's type space, and denote $\partial_{i} \Xi \equiv\left\{\mu \in \Xi: \mu_{i}=\mu_{i}\right\}$ as the set of $i$ th boundary types. Similar notations are used for the $n$-asset case. Also, $V(\mu, \alpha, \mathbf{p}(\overline{\alpha)})$ is often simply written as $V(\alpha)$.

\section{A.1 Symmetry result in Section 2.2}

Recall that $\mathcal{E}$ is the set of equilibrium selling strategies. Focus on the behavior of $\mathbf{p}$ on int $\mathcal{E}$, which is the interior of $\mathcal{E}$. Fix an agent type $\mu$ first. I denote $V(\mu, \alpha, \mathbf{p}(\alpha))$ as $V(\alpha ; \mu)$ without the risk of 
confusion. The gradient of Equation (2) evaluated at $\alpha$ is

$$
V_{i}(\alpha ; \mu) \equiv \frac{\partial V(\alpha ; \mu)}{\partial \alpha_{i}}=p^{(i)}-\mu_{i}+\sum_{j=1}^{2} \alpha_{j} p_{i}^{(j)}+r \sum_{j=1}^{2}\left(1-\alpha_{j}\right) \sigma_{i j}, \quad i=1,2 .
$$

Equation (FOC) in Section 2.2 essentially evaluates $V_{i}(\alpha ; \mu)$ at the agent's optimal strategy $\alpha^{*}$, which should be 0 due to optimality. Now consider $V_{i}(\widehat{\alpha} ; \mu)$ for $\forall \widehat{\alpha} \in i n t \mathcal{E}$. Since there exists some $\widehat{\mu} \neq \mu$ who takes $\widehat{\alpha}$, the agent $\widehat{\mu}$ 's FOC requires that $\sum_{j=1}^{2} \widehat{\alpha}_{j} p_{i}^{(j)}(\widehat{\alpha})+r \sum_{j=1}^{2}\left(1-\widehat{\alpha}_{j}\right) \sigma_{i j}=0$. Therefore, I have $V_{i}(\widehat{\alpha})=p^{(i)}(\widehat{\alpha})-\mu_{i}$ for all $\widehat{\alpha} \in i n t \mathcal{E}$. Differentiating this equation once more yields

$$
H(\alpha) \equiv \frac{\partial^{2} V}{\partial \alpha \partial \alpha^{\prime}}=\frac{d \mathbf{p}(\alpha)}{d \alpha^{\prime}} .
$$

In words, the Hessian matrix of the agent's value function $V$ equals the Jacobian matrix of the equilibrium pricing system $\mathbf{p}$. This result is useful in proving the concavity of $V$ later on. Finally, the symmetry of the Hessian matrix implies the symmetry result in the main text. This symmetry property also holds for the $n$-asset case, i.e., $p_{i}^{(j)}=p_{j}^{(i)}$ for all $i \neq j$.

\section{A.2 Proof of Proposition 1}

It is easy to check $\mathbf{p} \in \mathbf{C}^{1}$, i.e., continuously differentiable despite different functional forms on $\mathcal{A}_{i}$. I first show the strict concavity of $V$. Since $\frac{\partial^{2} V(\alpha)}{\partial \alpha \partial \alpha^{\prime}}=\frac{d \mathbf{p}(\alpha)}{d \alpha^{\prime}}$, it is easy to check that on $\mathcal{A}_{1}$,

$$
H=r\left[\begin{array}{rr}
\sigma_{11}\left(1-\frac{1}{\alpha_{1}}\right)-\sigma_{12} \frac{\alpha_{1}-\alpha_{2}}{\alpha_{1}^{2}} & \sigma_{12}\left(1-\frac{1}{\alpha_{1}}\right) \\
\sigma_{12}\left(1-\frac{1}{\alpha_{1}}\right) & \sigma_{22}\left(1-\frac{1}{\alpha_{2}}\right)
\end{array}\right],
$$

which is negative-definite (except on the boundary point $(1,1)$; but it has no bite on the strict concavity of $V$ ). Similar results hold for $\mathcal{A}_{2}$. Therefore $V$ is strictly concave on $\mathcal{A}_{i}$. Because $\mathbf{p} \in \mathbf{C}^{1}$, and $V \in \mathbf{C}^{1}$, which implies that, on any line crossing the diagonal, $V$ has strictly decreasing derivatives. Therefore, $V$ is strictly concave on the entire domain $\mathcal{A}$. Furthermore, it implies that each agent $\mu=\mathbf{p}(\alpha)$ has a unique optimal selling strategy $\alpha$; therefore, if $\mu \neq \mu^{\prime}$, their equilibrium strategies have to be different (otherwise market consistency is violated). Hence $\mathbf{p}$ is an equilibrium pricing system for a separating equilibrium.

To verify the intuitive criterion for this equilibrium, I use the definition in Game Theory by Fudenberg and Tirole (1991, p. 448). For simplicity, I imagine a Bertrand-type competition between $m$ identical risk-neutral investors ( $m \geq 2$ ), and only consider investors' equilibrium response given any possible belief system $\psi$ as in Microeconomic Theory by Mas-Colell, Whinston, and Green (1995, p. 169). Given any $0<\alpha \in \mathcal{A}$, the best response from any investor is $B R(\Xi, \alpha)=\Xi$, simply because $\mathbb{E}[\mu \mid \psi(\alpha)$.] could be any element in $\Xi$ if all reasonable beliefs $\psi$ are allowed. Since $\mathbf{p} \in$ $B R(\Xi, \alpha) \in \Xi$ could approach $+\infty$ in any entry, $J(\alpha) \equiv\left\{\mu: V^{*}(\mu)>\sup _{\mathbf{p} \in B R(\Xi, \alpha)} V(\mu, \alpha, \mathbf{p})\right\}=\varnothing$ for any $\alpha$. As a result, $B R(\Xi \backslash J(\alpha), \alpha)=\Xi$; then for any $\mu^{\prime}$ and her equilibrium strategy $\alpha^{\prime}$, $V^{*}\left(\mu^{\prime}\right)=V\left(\mu^{\prime}, \alpha^{\prime}, \mu^{\prime}\right) \geq \min _{\mathbf{p} \in \Xi} V\left(\mu^{\prime}, \alpha^{\prime}, \mathbf{p}\right)$. Finally, Pareto efficiency follows from the fact that now the one-dimensional types in $\partial_{i} \Xi$ behave as if their $i$ th asset is observable. Applying the same discretization method in the Corollary 1 of Grinblatt and Hwang (1989), it is not difficult to show that under this unidimensional type space and multidimensional signaling space setup, the standard LP result, which is preserved in this equilibrium, is the Pareto-dominant separating schedule. In other words, this equilibrium is Pareto-efficient for $\partial_{i} \Xi$ relative to all separating equilibria. Because of smoothness, once the boundary conditions are determined, the PDE leads to the pricing system obtained in the main text. 


\section{A.3 Proof of Proposition 2}

Consider $\mathcal{A}_{1}^{r}$; it is not difficult to verify that the Hessian matrix is

$$
H=r\left[\begin{array}{cc}
\sigma_{11}\left(1-\frac{1+\theta_{1}}{\alpha_{1}+\theta_{1} \alpha_{2}}\right) & \sigma_{12}\left(1-\frac{1+\theta_{1}}{\alpha_{1}+\theta_{1} \alpha_{2}}\right) \\
\sigma_{12}\left(1-\frac{1+\theta_{1}}{\alpha_{1}+\theta_{1} \alpha_{2}}\right) & \sigma_{22}\left(1-\frac{1-\rho^{2}}{\alpha_{2}}-\frac{\rho^{2}\left(1+\theta_{1}\right)}{\alpha_{1}+\theta_{1} \alpha_{2}}\right)
\end{array}\right],
$$

and note that $1-\frac{1-\rho^{2}}{\alpha_{2}}-\rho^{2} \frac{1+\theta_{1}}{\alpha_{1}+\theta_{1} \alpha_{2}}<1-\frac{1+\theta_{1}}{\alpha_{1}+\theta_{1} \alpha_{2}}<0$ on int $\mathcal{A}_{1}^{r}$. This shows that $H$ is negativedefinite on int $\mathcal{A}_{1}^{r}$, and similar results hold for int $\mathcal{A}_{2}^{r}$. To show that $V(\alpha)$ is strictly concave on $\mathcal{E}^{r}$, one could use the argument in Proposition 1. Similar arguments in Proposition 1 show that the separating equilibrium satisfies the intuitive criterion, and it is Pareto-efficient relative to other (smooth) separating equilibria.

\section{A.4 Proof of Proposition 3}

The first and second claims follow from a direct calculation of $\mathbf{p}(\cdot)$. The proof of $\frac{\partial p^{(i)}}{\partial \rho}>0$ needs a bit of explanation. The least obvious case is $\frac{\partial p^{r(1)}}{\partial \rho}$ on $\mathcal{A}_{2}^{r}$ when $\rho<0$. For simplicity, setting $r \sigma_{11}=r \sigma_{22}=1$ and $\underline{\mu_{1}}=0$, then on $\mathcal{A}_{2}^{r}$ I have (note that $\theta_{1}=\theta_{2}=\rho$ )

$$
p^{r(1)}(\alpha)=\alpha_{1}-\ln \alpha_{1}-1-\rho^{2}\left(\ln \frac{\alpha_{2}+\rho \alpha_{1}}{1+\rho}-\ln \alpha_{1}\right)+\rho\left(\alpha_{2}-\ln \frac{\alpha_{2}+\rho \alpha_{1}}{1+\rho}-1\right),
$$

and

$$
\begin{aligned}
\frac{\partial p^{r(1)}}{\partial \rho} & =-\rho(1+\rho)\left(\frac{\alpha_{1}}{\alpha_{2}+\rho \alpha_{1}}-\frac{1}{1+\rho}\right)-2 \rho \ln \frac{\alpha_{2}+\rho \alpha_{1}}{(1+\rho) \alpha_{1}}-\ln \frac{\alpha_{2}+\rho \alpha_{1}}{1+\rho}+\alpha_{2}-1 \\
& =\frac{\rho\left(\alpha_{2}-\alpha_{1}\right)}{\alpha_{2}+\rho \alpha_{1}}-2 \rho \ln \frac{\alpha_{2}+\rho \alpha_{1}}{(1+\rho) \alpha_{1}}-\ln \frac{\alpha_{2}+\rho \alpha_{1}}{1+\rho}+\alpha_{2}-1 .
\end{aligned}
$$

Since $\frac{\alpha_{2}+\rho \alpha_{1}}{1+\rho} \in(0,1), \frac{\alpha_{2}+\rho \alpha_{1}}{1+\rho}-\ln \frac{\alpha_{2}+\rho \alpha_{1}}{1+\rho}-1>0$, it suffices to show the following term is positive:

$$
\begin{aligned}
& \frac{\rho\left(\alpha_{2}-\alpha_{1}\right)}{\alpha_{2}+\rho \alpha_{1}}-2 \rho \ln \frac{\alpha_{2}+\rho \alpha_{1}}{(1+\rho) \alpha_{1}}+\alpha_{2}-\frac{\alpha_{2}+\rho \alpha_{1}}{1+\rho} \\
= & \rho\left(\alpha_{2}-\alpha_{1}\right)\left(\frac{1}{\alpha_{2}+\rho \alpha_{1}}+\frac{1}{1+\rho}\right)-2 \rho \ln \frac{\alpha_{2}+\rho \alpha_{1}}{(1+\rho) \alpha_{1}} \\
> & 2 \rho \frac{\alpha_{2}-\alpha_{1}}{\alpha_{2}+\rho \alpha_{1}}-2 \rho \ln \frac{\alpha_{2}+\rho \alpha_{1}}{(1+\rho) \alpha_{1}}
\end{aligned}
$$

where I use $\alpha_{2}+\rho \alpha_{1} \leq 1+\rho$ and $\rho\left(\alpha_{2}-\alpha_{1}\right)<0$ for $\alpha \in \mathcal{A}_{2}^{r}$. Let $\beta=\frac{(1+\rho) \alpha_{1}}{\alpha_{2}+\rho \alpha_{1}} \in(0,1)$; since $\rho<0$, I need to show that $\frac{\alpha_{2}-\alpha_{1}}{\alpha_{2}+\rho \alpha_{1}}+\ln \beta<0$. Now since $\frac{\alpha_{2}-\alpha_{1}}{\alpha_{2}+\rho \alpha_{1}}=1-\beta$, and $-\beta+\ln \beta+1<0$, I get the desired result. The third result follows from the envelope theorem. I need some extra work for $\frac{\partial V}{\partial \rho}$. When $\rho>0$, one can check that when $\alpha \in \mathcal{A}_{1}$ (i.e., $\alpha_{1} \geq \alpha_{2}$ ),

$$
\begin{aligned}
\frac{\partial V}{\partial \rho} & =\alpha_{1} \frac{\partial p^{(1)}}{\partial \rho}+\alpha_{2} \frac{\partial p^{(2)}}{\partial \rho}-r \sigma_{1} \sigma_{2}\left(1-\alpha_{1}\right)\left(1-\alpha_{2}\right) \\
& =-\left(\alpha_{1}+\alpha_{2}\right) \ln \alpha_{1}-\left(1+\alpha_{1}\right)\left(1-\alpha_{2}\right) .
\end{aligned}
$$

which is increasing in $\alpha_{2}$. Therefore to verify $\frac{\partial V}{\partial \rho}<0$, it suffices to show $m\left(\alpha_{1}\right) \equiv-2 \alpha_{1} \ln \alpha_{1}+$ $\alpha_{1}^{2}-1<0$ for $\alpha_{1} \in(0,1)$. But it is easy to check that $m(1)=0$, and $m^{\prime}\left(\alpha_{1}\right)=2\left(\alpha_{1}-\ln \alpha_{1}-1\right)>$ 0 , which implies the claim I need. The results for $\alpha \in \mathcal{A}_{2}$ and negative correlation case follow similarly (when $\rho<0$, I need a trick similar to the proof for Proposition 5: consider the change of $\frac{\partial V}{\partial \rho}$ along the lines parallel to $\left.A_{1}^{r}\right)$. 


\section{A.5 Proof of Proposition 4}

Note that given $\mathbf{p}^{i r}(\cdot)$, the optimal strategy of any type of agent must lie on $\mathcal{E}^{i r}$. Once I restrict the agent's selling strategy to be within $\mathcal{E}^{i r} \subset \mathcal{A}_{1}^{r}, V(\alpha)$ is strictly concave on $\mathcal{E}^{i r}$, and her optimal selling strategy is unique and type-revealing by the results obtained in the regular case in Proposition 2 (recall that $V(\alpha)$ is strictly concave on $\mathcal{A}_{1}^{r}$ ). Therefore $\mathbf{p}^{i r}(\cdot)$ delivers a separating equilibrium for the irregular case. The verification of intuitive criterion and Pareto efficiency follows similarly as the argument in Proposition 1.

\section{A.6 Property of $A_{2}^{i r}$ in Section 5.1}

Consider any characteristic line $L \in \mathcal{A}_{1}^{r}$. Along $L$ toward the origin $\mathbf{O}$, the proof for Lemma 1 (see below) shows that $p^{r(2)}$ is greater than $\mu_{2}$ initially, but finally drops below $\mu_{2}$ when it is sufficiently close to $\mathbf{O}$ (see Figure 4 ). Therefore I define the curve $A_{2}^{i r} \equiv\left\{\alpha \in \mathcal{A}_{1}^{r}: p^{\overline{r(2)}}(\alpha)=\underline{\mu_{2}}\right\}$ as the new boundary for $\mathcal{E}^{i r}$, and in equilibrium all types in $\partial \Xi_{2}$ lie on this curve. The following lemma states the properties of this curve. When $\theta_{2}=-1, A_{2}^{i r}=A_{2}^{r}$ is just the diagonal line $\left\{\alpha \in \mathcal{A}: \alpha_{1}=\alpha_{2}\right\}$.

Lemma 1. The curve $A_{2}^{i r}$, with $(1,1)$ and $(0,0)$ as its upper-and lower-ending points, respectively, lies between $A_{2}^{r}$ and the diagonal line $\left\{\alpha \in \mathcal{A}: \alpha_{1}=\alpha_{2}\right\}$.

Proof. First, $(1,1) \in A_{2}^{i r}$; later on I ignore this upper-ending point. Given any $L \in \mathcal{A}_{1}^{r}$, decompose $L$ into $L^{\prime} \cup L^{\prime \prime}$ where $L^{\prime}\left(L^{\prime \prime}\right)$ is on the right- (left-) hand side of $A_{2}^{r}$ (see Figure 4). Rewrite the PDE satisfied by $p^{r(2)}$ as

$$
-\left(\alpha_{1} p_{1}^{r(2)}+\alpha_{2} p_{2}^{r(2)}\right)=r \sigma_{22}\left[\alpha_{2}^{F B}\left(\alpha_{1}\right)-\alpha_{2}\right]
$$

which describes the marginal increment of $p^{r(2)}$ along $L$ toward $\mathbf{O}$. Since on $L^{\prime}\left(L^{\prime \prime}\right)$ I have $\alpha_{2}^{F B}\left(\alpha_{1}\right)-\alpha_{2} \geq(<) 0, p^{r(2)}$ increases first on $L^{\prime}$ then decreases on $L^{\prime \prime}$, and achieves maximum on $A_{2}^{r}$. I can use this fact to prove that $p^{r(2)}>\underline{\mu_{2}}$ on $A_{2}^{r}$. To show this, note that on $A_{1}^{r}, p^{r(2)}(\alpha)=\underline{\mu_{2}}+$ $r\left(1-\rho^{2}\right) \sigma_{22}\left(\alpha_{2}-\ln \alpha_{2}-1\right)>\mu_{2}$, and, for any point $\alpha^{\prime} \in A_{2}^{r}$, I can find a transport path from a particular point sitting on $A_{1}^{r}$. Because $p^{r(2)}$ achieves maximum on $A_{2}^{r}$, the claim follows. Next, it is not difficult to see that along the diagonal, $p^{r(2)}(\alpha, \alpha)=\underline{\mu_{2}}+r \sigma_{22}\left(1+\theta_{2}\right)(\alpha-\ln \alpha-1) \leq \underline{\mu_{2}}$ since $1+\theta_{2} \leq 0$. According to the continuity of $p^{r(2)}(\alpha), A_{2}^{i r}$ must be between $A_{2}^{r}$ and the diagonal line. Finally, I show that $\lim _{y \rightarrow 0^{+}} \alpha_{1}(y)=0$, or $(0,0)$ is the lower-ending point of $A_{2}^{i r}$. It suffices to show that the lim sup is 0 . For a sequence $\left\{y_{n}\right\} \rightarrow 0$, let the lim sup of $\alpha_{1}\left(y_{n}\right)$ be $\bar{\alpha}_{1}$. Suppose $\bar{\alpha}_{1}>0$; I can choose subsequence $\left\{y_{n^{\prime}}\right\}$ so that $\alpha_{1}\left(y_{n^{\prime}}\right) \geq \bar{\alpha}_{1} / 2>0$ for all $n^{\prime}$. But $p^{r(2)}\left(\alpha_{1}\left(y_{n^{\prime}}\right), y_{n^{\prime}}\right) \geq p^{r(2)}\left(\bar{\alpha}_{1} / 2, y_{n^{\prime}}\right)$, and the latter could be arbitrarily large for $y_{n^{\prime}}$ close enough to 0 . This contradicts the equality $p^{r(2)}\left(\alpha_{1}\left(y_{n^{\prime}}\right), y_{n^{\prime}}\right)=\underline{\mu_{2}}$.

\section{A.7 Proof of Proposition 5}

It is easier to start with the case $\rho<0$. Suppose the assets are regular, and denote the equilibrium selling strategy under the separate sale as $\alpha=\left(\alpha_{1}, \alpha_{2}\right)$. We can view $\alpha$ as a function of $\alpha=\left(\alpha_{1}, \alpha_{2}\right)$ as implicitly defined by the market consistency condition:

$$
\alpha(\alpha)-\ln \alpha(\alpha)-1=\frac{p^{r(1)}(\alpha)-\underline{\mu_{1}}+p^{r(2)}(\alpha)-\underline{\mu_{2}}}{r\left(\sigma_{11}+2 \sigma_{12}+\sigma_{22}\right)} .
$$

It is clear that when $\alpha_{1}=\alpha_{2}$, which holds if $\frac{\mu_{1}-\underline{\mu_{1}}}{\sigma_{11}+\sigma_{12}}=\frac{\mu_{2}-\underline{\mu_{2}}}{\sigma_{22}+\sigma_{12}}, \alpha$ has to be equal to them as well; hence, in this case we have the equivalence between the separate sale and the pooled sale. Now consider the domain $\mathcal{A}_{1}^{r}$. Define

$$
Q(\alpha) \equiv(1-\alpha(\alpha))^{2}-\frac{\left(1-\alpha_{1}\right)^{2} \sigma_{11}+2\left(1-\alpha_{1}\right)\left(1-\alpha_{2}\right) \sigma_{12}+\left(1-\alpha_{2}\right)^{2} \sigma_{22}}{\left(\sigma_{11}+2 \sigma_{12}+\sigma_{22}\right)} ;
$$


since $Q(\alpha)=0$ when $\alpha_{1}=\alpha_{2}$, and now $\alpha_{2}<\alpha_{1}$, it is sufficient to show that $Q_{1}(\alpha) \equiv \frac{\partial Q}{\partial \alpha_{1}}>0$. Direct calculation and the implicit function theorem yields $\left(Q_{1}(\alpha)=M(\alpha) \cdot\left[1-\frac{\left(1+\theta_{1}\right) \alpha(\alpha)}{\alpha_{1}+\theta_{1} \alpha_{2}}\right]\right.$, where $M(\alpha)$ is positive)

$$
\operatorname{sign}\left(Q_{1}(\alpha)\right)=\operatorname{sign}\left(\frac{\alpha_{1}+\theta_{1} \alpha_{2}}{1+\theta_{1}}-\alpha(\alpha)\right)
$$

Let $\delta^{-}(\alpha) \equiv \frac{\alpha_{1}+\theta_{1} \alpha_{2}}{1+\theta_{1}}$ where "-" indicates the negative correlation. To show that $\delta^{-}(\alpha)>\alpha(\alpha)$, it suffices to show that (note that $x-\ln x-1$ is strictly decreasing)

$$
\delta^{-}(\alpha)-\ln \delta^{-}(\alpha)-1>\frac{p^{r(1)}(\alpha)-\underline{\mu_{1}}+p^{r(2)}(\alpha)-\underline{\mu_{2}}}{r\left(\sigma_{11}+2 \sigma_{12}+\sigma_{22}\right)} .
$$

Plug in $\mathbf{p}^{r}$ and rearrange to show that $\sigma_{22}\left(1-\rho^{2}\right)\left[\ln \frac{\delta^{-}(\alpha)}{\alpha_{2}}-\frac{\alpha_{1}-\alpha_{2}}{1+\theta_{1}}\right] \geq 0$. But this holds trivially, since for $\ln \frac{\delta^{-}(\alpha)}{\alpha_{2}}-\frac{\alpha_{1}-\alpha_{2}}{1+\theta_{1}}$, when $\alpha_{1}=\alpha_{2}$ it is 0 , and its derivative w.r.t $\alpha_{1}$ is $\frac{1}{\alpha_{1}+\theta_{1} \alpha_{2}}-\frac{1}{1+\theta_{1}}>0$ for $\alpha \in$ int $\mathcal{A}_{1}^{\alpha_{2}}$. I can carry out the same argument for $\mathcal{A}_{2}^{r}$; this concludes the proof for the regular case with $\rho<0$.

If the assets are irregular, pick the corresponding domain and apply the argument above. Note that in the equilibrium of separate sales I cannot have equal fractions, hence the separate sale always dominates the pooled sale.

The positive correlation case is more involved. Replace $\mathbf{p}^{r}$ by $\mathbf{p}$ and define $Q(\alpha)$ as before. Consider $\mathcal{A}_{1}$ first; and instead of showing $Q_{1}(\alpha)>0$ as before, I show $Q_{2}(\alpha)<0$. I have

$$
\operatorname{sign}\left(Q_{2}(\alpha)\right)=\frac{1-\alpha_{2}+\theta_{2}\left(1-\alpha_{1}\right)}{\frac{1}{\alpha_{2}}-1+\theta_{2}\left(\frac{1}{\alpha_{1}}-1\right)}-\alpha(\alpha) \text {. }
$$

Let $\delta^{+}(\alpha) \equiv \frac{1-\alpha_{2}+\theta_{2}\left(1-\alpha_{1}\right)}{\frac{1}{\alpha_{2}}-1+\theta_{2}\left(\frac{1}{\alpha_{1}}-1\right)}$, then it suffices to show $\delta^{+}(\alpha)<\alpha(\alpha)$. Let $\gamma=\frac{\sigma_{11}}{\sigma_{22}}$; the strategy is to show that (note that $\alpha_{2}-\ln \alpha_{2}-1 \geq \alpha_{2}-\ln \alpha_{1}-\frac{\alpha_{2}}{\alpha_{1}}$ )

$$
\begin{aligned}
\delta^{+}(\alpha)-\ln \delta^{+}(\alpha)-1 & >\frac{\left(\gamma+\theta_{2}\right)\left(\alpha_{1}-\ln \alpha_{1}-1\right)+\left(\theta_{2}+1\right)\left(\alpha_{2}-\ln \alpha_{2}-1\right)}{\gamma+2 \theta_{2}+1} \\
& \geq \frac{\left(\gamma+\theta_{2}\right)\left(\alpha_{1}-\ln \alpha_{1}-1\right)+\theta_{2}\left(\alpha_{2}-\ln \alpha_{1}-\frac{\alpha_{2}}{\alpha_{1}}\right)+\left(\alpha_{2}-\ln \alpha_{2}-1\right)}{\gamma+2 \theta_{2}+1} .
\end{aligned}
$$

Note also that the second line is just $\alpha(\alpha)-\ln \alpha(\alpha)-1$ on $\mathcal{A}_{1}$. Define

$$
R(\alpha) \equiv \delta^{+}(\alpha)-\ln \delta^{+}(\alpha)-1-\frac{\left(\gamma+\theta_{2}\right)\left(\alpha_{1}-\ln \alpha_{1}-1\right)+\left(\theta_{2}+1\right)\left(\alpha_{2}-\ln \alpha_{2}-1\right)}{\gamma+2 \theta_{2}+1} ;
$$

I want to show $R(\alpha)>0$ for $\alpha_{2}<\alpha_{1}$. (Note that $R(\alpha)=0$ when $\alpha_{1}=\alpha_{2}$.)

Let $b \equiv \frac{\alpha_{2}+\theta_{2} \alpha_{1}}{1+\theta_{2}} \in(0,1)$, and I can define a parameterized line $\alpha(t)$ for $t \geq 0$, where $\left\{\begin{array}{l}\alpha_{1}(t)=b+t \\ \alpha_{2}(t)=b-\theta_{2} t\end{array}\right.$. It starts from $(b, b)$ and reaches $\alpha$ when $t=\frac{\alpha_{1}-\alpha_{2}}{\theta_{2}+1}>0$. 
Now, $R(\alpha)=R(\alpha)-R(b, b)=\int_{0}^{\frac{\alpha_{1}-\alpha_{2}}{\theta_{2}+1}} \frac{d R(\alpha(t))}{d t} d t$, so it is sufficient to show that $\frac{d R(\alpha(t))}{d t}=$ $R_{1}(\alpha(t))-\theta_{2} R_{2}(\alpha(t))>0$. I have

$$
\begin{aligned}
\frac{d R(\alpha(t))}{d t}= & \frac{\theta_{2}\left(\alpha_{2}+\frac{1}{\alpha_{2}}-2+\theta_{2}\left(\alpha_{1}+\frac{1}{\alpha_{1}}-2\right)\right)\left(\frac{1}{\alpha_{2}^{2}}-\frac{1}{\alpha_{1}^{2}}\right)}{\left(\frac{1}{\alpha_{2}}-1+\theta_{2}\left(\frac{1}{\alpha_{1}}-1\right)\right)^{2}} \\
& +\frac{\theta_{2}\left(\frac{1}{\alpha_{1}}-\frac{1}{\alpha_{2}}\right)+\gamma\left(\frac{1}{\alpha_{1}}-1\right)-\theta_{2}^{2}\left(\frac{1}{\alpha_{2}}-1\right)}{\gamma+2 \theta_{2}+1} \\
> & \theta_{2}\left(\frac{1}{\alpha_{2}}-\frac{1}{\alpha_{1}}\right)\left(\frac{\left(\alpha_{2}+\frac{1}{\alpha_{2}}-2+\theta_{2}\left(\alpha_{1}+\frac{1}{\alpha_{1}}-2\right)\right)\left(\frac{1}{\alpha_{1}}+\frac{1}{\alpha_{2}}\right)}{\left(\frac{1}{\alpha_{2}}-1+\theta_{2}\left(\frac{1}{\alpha_{1}}-1\right)\right)^{2}}-\frac{\theta_{2}+1}{\gamma+2 \theta_{2}+1}\right),
\end{aligned}
$$

hence it amounts to showing that the second bracket is positive. To show this, let $u \equiv \frac{1}{\alpha_{1}}-1 \geq$ $0, v \equiv \frac{1}{\alpha_{2}}-1 \geq 0 ;$ since $\gamma^{2}>\theta_{2}^{2}=\frac{\sigma_{11 \rho^{2}}}{\sigma_{22}}$, I have

$$
\begin{gathered}
\left(v^{2}+\frac{u+1}{v+1} v^{2}+\theta_{2} \frac{u+1}{v+1} u^{2}+\theta_{2} u^{2}\right)\left(\gamma+2 \theta_{2}+1\right)-\left(\theta_{2}+1\right)\left(v+\theta_{2} u\right)^{2} \\
\geq \theta_{2} \frac{u+1}{v+1} u^{2}+\theta_{2} u^{2}+2 \theta_{2} \frac{u+1}{v+1} v^{2}+\gamma u^{2}+\gamma \theta_{2} \frac{u+1}{v+1} u^{2}>0 .
\end{gathered}
$$

To recap, when $\alpha \in \mathcal{A}_{1}, R(\alpha)>0$; then $\delta^{+}(\alpha)<\alpha(\alpha)$, or $Q_{2}(\alpha)<0$; finally $Q(\alpha)>0$, which implies the dominance of separate sale. Similarly, I can show that it holds for $\alpha \in \mathcal{A}_{2}$.

\section{A.8 Detailed examples for Section 5.3}

\section{A.8.1 Equilibrium pricing system for the positively correlated $\boldsymbol{n}$-asset case.}

I can solve the $n$-asset case recursively when all correlations are positive. Let $\mathbf{n} \equiv\{1,2, \ldots, n\}$. When $\sigma_{i j} \geq 0$ for $\forall i, j \in \mathbf{n}, B C$ implies that the $i$ th boundary-type agent will always set $\alpha_{i}=1$. Once the agent keeps zero inventory for asset $i$, I essentially reduce an $n$-dimensional problem to an $(n-1)$-dimensional one. By induction, I have a simple formula for the $n$-dimensional equilibrium pricing schedule:

$$
p^{(i)}(\alpha)=\underline{\mu_{i}}+r \sum_{j \in \overline{\mathcal{I}}_{i}(\alpha)} \sigma_{i j}\left(\alpha_{j}-\ln \alpha_{j}-1\right)+r \sum_{j \in \underline{\mathcal{I}}_{i}(\alpha)} \sigma_{i j}\left(\alpha_{j}-\ln \alpha_{i}-\frac{\alpha_{j}}{\alpha_{i}}\right),
$$

where $\overline{\mathcal{I}}_{i}(\alpha) \equiv\left\{k \in \mathbf{n}: \alpha_{k} \geq \alpha_{i}\right\}$, and $\underline{\mathcal{I}}_{i}(\alpha) \equiv \mathbf{n} \backslash \overline{\mathcal{I}}_{i}(\alpha)$. One can check that $p^{(i)}$ has continuous derivatives despite the different functional forms across various regions. Finally, similar to the proof for 2-asset case, I can show that $V(\mu, \alpha, \mathbf{p}(\alpha))$ is strictly concave on $(0,1]^{n}$, and therefore $\mathbf{p}(\cdot)$ is a separating equilibrium. For details, see He (2005).

A.8.2 Example of equilibrium construction for a three-asset case with a general covariance matrix. This example illustrates how to construct an equilibrium pricing system under a general variance structure. For simplicity I assume $\mu=(0,0,0)$ and $r=1$. I consider the covariance matrix

$$
\left[\begin{array}{rrr}
1 & \frac{1}{2} & 0 \\
\frac{1}{2} & 1 & -\frac{1}{2} \\
0 & -\frac{1}{2} & 1
\end{array}\right]
$$


as shown later, this case demonstrates all main issues in deriving the equilibrium pricing system. In this example, asset 1 and asset 2 are positively correlated, while asset 2 and asset 3 are negatively correlated. Asset 1 and asset 3, however, are independent. The new insight comes from the asset 2, because it serves two opposite "hedging" roles for the other two assets.

One can find pricing formulas for boundaries of $\mathcal{E} \subset(0,1]^{3}$ (i.e., the surfaces where the boundary agents sit) by invoking $B C$. First consider $\partial \Xi_{1}$; because asset 1 has nonnegative covariances with both assets, $\alpha_{1}^{F B}\left(\alpha_{2}, \alpha_{3}\right)=1$ always. Therefore, $\partial \Xi_{1}$ types still employ the strategy with $\left\{\alpha: \alpha_{1}=1\right\}$, and the pricing system for asset 2 and 3 (negatively correlated) is simply the system (6) obtained in Section 3.2.2, with $\rho=-\frac{1}{2}$. Of course $p^{(1)}(\alpha)=0$ for $\left\{\alpha: \alpha_{1}=1\right\}$.

Now consider $\partial \Xi_{3}$. Given $\alpha_{1}$ and $\alpha_{2}$, the conditional first-best selling amount is $\alpha_{3}^{F B}\left(\alpha_{1}, \alpha_{2}\right)=$ $\frac{1}{2}+\frac{\alpha_{2}}{2} \in[0,1]$. Therefore, the type-3 boundary for $\mathcal{E}$ is a plane $\left\{\alpha:\left(\alpha_{1}, \alpha_{2}, \frac{1}{2}+\frac{\alpha_{2}}{2}\right)\right\}$. On this plane, the problem is a 2 -asset case with a positive correlation; but the covariance matrix between these two assets becomes $\left[\begin{array}{cc}1 & 1 / 2 \\ 1 / 2 & 3 / 4\end{array}\right]$ due to the optimal hedging from asset 3 . Therefore, the threedimensional pricing system on this plane is as in Equation (5), and $p^{(3)}(\alpha)=0$.

Finally, I consider $\partial \Xi_{2}$. Because the retention of asset 2 is beneficial for asset 3 but harmful in the view of asset 1 , this becomes the most intriguing case that involves a kinked surface on the boundary. Given $\alpha_{1}$ and $\alpha_{2}$, the unconstrained optimal solution is $\alpha_{2}^{F B}\left(\alpha_{1}, \alpha_{3}\right)=1+\frac{\alpha_{3}-\alpha_{1}}{2}$. However, since I require $\alpha_{2}^{F B} \in[0,1]$, as a result:

$$
\alpha_{2}^{F B}\left(\alpha_{1}, \alpha_{3}\right)=\left\{\begin{array}{cl}
1 & \text { if } \alpha_{3}>\alpha_{1} \\
1+\frac{\alpha_{3}-\alpha_{1}}{2} & \text { if } \alpha_{3} \leq \alpha_{1}
\end{array}\right.
$$

Intuitively, when the agent has a large position on asset 1 , which pushes her to sell asset 2, the short-sale constraint might bind. On the other hand, if she retains a large amount of asset 3 , which calls for hedging from asset 2 , she is free to do so. This complication delivers a kinked surface for the type-2 boundary $\left\{\alpha:\left(\alpha_{1}, \alpha_{2}^{F B}\left(\alpha_{1}, \alpha_{3}\right), \alpha_{3}\right)\right\}$. When $\alpha_{3}>\alpha_{1}$, the agent sells her entire asset 2; and because asset 1 and 3 are independent, I have the LP results for both assets. When $\alpha_{3} \leq \alpha_{1}$, these two assets have an effective variance matrix $\left[\begin{array}{ll}3 / 4 & 1 / 4 \\ 1 / 4 & 3 / 4\end{array}\right]$ (asset 3 needs retention from asset 1 , which has positive correlation with asset 2 , therefore, these two independent assets look as if they are positively correlated), and the pricing system is the one as in Equation (5).

Once these boundary pricing systems are ready, the last step is to obtain the interior function value by integration. Nevertheless, the tedious line integration cannot bring any new economic insight.

\section{A.9 Detailed examples for Section 5.4}

A.9.1 Constructing a Pareto-inefficient separating equilibrium. For illustrative purpose, consider the case $n=2$ and $\rho>0$, with $r \sigma_{11}=r \sigma_{22}=1$, and $\underline{\mu}=(0,0)$. I try to construct an equilibrium schedule where agents with $\mu_{1}$ optimally choose selling strategies $\alpha_{1}<1$. Specifically, for any small $k>0$, define the potential boundary $B^{k}$ as

$$
B^{k} \ni \alpha=\left\{\begin{array}{cl}
\left(1-\frac{x}{2}, 1-x\right) & \text { for } 0 \leq x<2 k \\
\left(1-2 k+\frac{x}{2}, 1-x\right) & \text { for } 2 k \leq x<4 k \\
(1,1-x) & \text { for } 4 k \leq x<1
\end{array}\right.
$$

In words, $B^{k}$ has a triangle dent (call the triangle $\overline{\mathcal{B}}^{k}$, which is the shaded area in Figure 5; the particular value (here 2) of the slope for the dent is inessential) relative to $A_{1}$. The set of equilibrium strategies $\mathcal{E}^{k}$ is $(0,1]^{2} \backslash \overline{\mathcal{B}}^{k}$, which includes $B^{k}$ as its boundary.

Now I derive the equilibrium pricing system $\mathbf{q}=\left(q^{(1)}, q^{(2)}\right)$ for this case. I restrict the analysis on $\mathcal{A}_{1}$ where $\alpha_{1} \geq \alpha_{2}$ (for $\mathcal{A}_{2}$ the pricing system $\mathbf{q}$ is just the one in Equation (5)). Consider those 


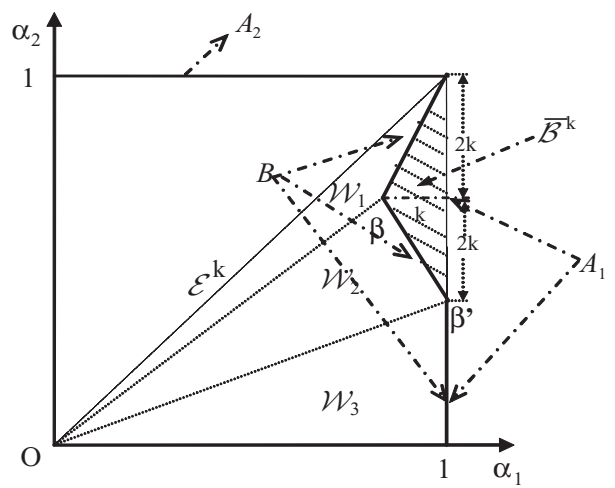

Figure 5

An example of inefficient separating equilibria that violate the $\mathrm{BC}$ assumption

In this equilibrium, $\partial \Xi_{1}$ types lie on the dented line $B$ instead of $A_{1}=\left\{\alpha \in \mathcal{A}: \alpha_{1}=1\right\}$, because of the penalty from the asset 2 pricing. As a result, $\overline{\mathcal{B}}^{k}$ is off-equilibrium.

types in $\partial \Xi_{1}$; for their asset 2 pricing, the unidimensional analysis yields

$q^{(2)}(\alpha)= \begin{cases}\frac{5+4 \rho}{4}\left(\alpha_{2}-\ln \alpha_{2}-1\right) & \text { for } 1-2 k<\alpha_{2} \leq 1 \\ q^{(2)}(\beta)+\frac{5-4 \rho}{4}\left(\alpha_{2}-\ln \frac{\alpha_{2}}{1-2 k}-1+2 k\right)-(2 \rho-1) k \ln \frac{\alpha_{2}}{1-2 k} & \text { for } 1-4 k<\alpha_{2} \leq 1-2 k \\ q^{(2)}\left(\beta^{\prime}\right)+\alpha_{2}-\ln \frac{\alpha_{2}}{1-4 k}-1+4 k & \text { for } 0<\alpha_{2} \leq 1-4 k\end{cases}$

for $\alpha \in B$, where $\beta$ and $\beta^{\prime}$ are the two kink points on the triangle shown in Figure 5. Of course $q^{(1)}(\alpha)=0$. As shown in Figure 5, I denote three regions-generated by two boundary characteristic lines-as $\mathcal{W}_{m}$ for $m=1,2,3$ on $\mathcal{A}_{1} \backslash \overline{\mathcal{B}}^{k}$. Using the same technique as before, I obtain

$$
\left\{\begin{array}{l}
q^{(1)}(\alpha)=-\left(\rho \alpha_{2}+\alpha_{1}\right)\left(\frac{1}{2 \alpha_{1}-\alpha_{2}}-1\right)-(1+\rho) \ln \left(2 \alpha_{1}-\alpha_{2}\right), \\
q^{(2)}(\alpha)=\frac{5+4 \rho}{4}\left(\frac{\alpha_{2}}{2 \alpha_{1}-\alpha_{2}}-\ln \frac{\alpha_{2}}{2 \alpha_{1}-\alpha_{2}}-1\right)-\left(\rho \alpha_{1}+\alpha_{2}\right)\left(\frac{1}{2 \alpha_{1}-\alpha_{2}}-1\right)-(1+\rho) \ln \left(2 \alpha_{1}-\alpha_{2}\right),
\end{array}\right.
$$

for $\alpha \in \mathcal{W}_{1}$, and

$$
\left\{\begin{array}{rl}
q^{(1)}(\alpha)= & -\left(\rho \alpha_{2}+\alpha_{1}\right)\left(\frac{3-4 k}{2 \alpha_{1}+\alpha_{2}}-1\right)+(1+\rho) \ln \frac{3-4 k}{2 \alpha_{1}+\alpha_{2}}, \\
q^{(2)}(\alpha)= & q^{(2)}(\beta)+\frac{5-4 \rho}{4}\left(\frac{\alpha_{2}(3-4 k)}{2 \alpha_{1}+\alpha_{2}}-\ln \frac{\alpha_{2}(3-4 k)}{2 \alpha_{1}+\alpha_{2}}-1+2 k+\ln (1-2 k)\right) \\
& -(2 \rho-1) k \ln \frac{\alpha_{2}(3-4 k)}{\left(2 \alpha_{1}+\alpha_{2}\right)(1-2 k)}-\left(\rho \alpha_{1}+\alpha_{2}\right)\left(\frac{3-4 k}{2 \alpha_{1}+\alpha_{2}}-1\right)+(1+\rho) \ln \frac{3-4 k}{2 \alpha_{1}+\alpha_{2}}
\end{array},\right.
$$

for $\alpha \in \mathcal{W}_{2}$, and

$$
\left\{\begin{array}{l}
q^{(1)}(\alpha)=-\left(\rho \alpha_{2}+\alpha_{1}\right)\left(\frac{1}{\alpha_{1}}-1\right)-(1+\rho) \ln \alpha_{1}, \\
q^{(2)}(\alpha)=q^{(2)}\left(\beta^{\prime}\right)-\ln \frac{\alpha_{2} / \alpha_{1}}{1-4 k}+4 k+\left(\rho \alpha_{1}+\alpha_{2}\right)-\rho-(1+\rho) \ln \alpha_{1},
\end{array}\right.
$$

for $\alpha \in \mathcal{W}_{3}$. For $\alpha \in \mathcal{A}_{2}$ the pricing system $\mathbf{q}$ is the same as in Equation (5), and $\mathbf{q}(\alpha)=(0,0)$ for $\alpha \in \overline{\mathcal{B}}^{k}$.

One can check that $\mathbf{q} \notin \mathbb{C}^{1}\left(\mathcal{E}^{k}\right)$ (along the boundary between $\mathcal{W}_{i}$ 's, the individual pricing functions in $\mathbf{q}$ have kinks); however, $V$, as a composite function of $\mathbf{q}$, is in fact smooth (both kinks from $q^{(i)}$ 's cancel each other). One can check that $H=\frac{\mathcal{D} \mathbf{q}}{\mathcal{D} \alpha}$ is negative-definite over all domains. 
Therefore if the agent is restricted in the domain $\mathcal{E}^{k}, \mathbf{q}$ still constitutes an equilibrium pricing system.

To show that the agent will not deviate to $\overline{\mathcal{B}}^{k}$, it suffices to consider the boundary types $(0, \mu) \in \partial \Xi_{1}$. The reason is that the interior types (with a better asset 1 ) have less incentives to sell more. Formally, for any interior type $\mu=\left(\mu_{1}, \mu_{2}\right)$ who chooses $\alpha$, and its corresponding boundary type $\mu^{\prime}=\left(0, \mu_{2}\right)$ who chooses $\alpha^{\prime}$, if the deviating point is $\gamma$ which necessarily has $\gamma_{1}=1$, then it is easy to check that $V(\mu, \gamma)-V\left(\mu, \alpha^{\prime}\right)<V\left(\mu^{\prime}, \gamma\right)-V\left(\mu^{\prime}, \alpha^{\prime}\right)$. But because $V(\mu, \alpha)>V\left(\mu, \alpha^{\prime}\right)$, we have $V(\mu, \gamma)-V(\mu, \alpha)<V\left(\mu^{\prime}, \gamma\right)-V\left(\mu^{\prime}, \alpha^{\prime}\right)$, i.e., a smaller deviation gain.

Now, for a sufficiently small $k$, I only have to consider $\mu$ close to 0 . Generally, in these separating equilibria, the type $(0, \mu)$ 's value is in the order of $\mu^{\frac{3}{2}}$ for small $\mu$. (To see this, if the agent is on $\mathcal{W}_{1}$, then $V=\mu-\frac{5+4 \rho}{8}(1-\alpha(\mu))^{2}$ and $\mu=\frac{5+4 \rho}{4}[\alpha(\mu)-\ln \alpha(\mu)-1]$. Now view $\mu(x)$ as a function of $x \equiv 1-\alpha \geq 0$, and the Taylor expansion around $\mu(0)=0$ yields the result. If the agent is on $\mathcal{W}_{2}$ the value incremental is in the same order.) However, since the value from deviating (to $(1, \gamma)$ ) for a small $\mu$ (she solves $\left.\max _{\gamma \in[1-4 k, 1]}(1-\gamma) \mu-\frac{1}{2}(1-\gamma)^{2}\right)$ is at most in the order of $\mu^{2}$, for any sufficiently small $k$ the resulting pricing system is indeed a separating equilibrium.

In this equilibrium, those types in $\partial \Xi_{1}$ hold certain positive positions of asset 1 , due to a substantial penalty imposed by $p^{(2)}(\cdot)$. The resulting continuum of equilibria (indexed by $k$ ) are less efficient by design. One can verify that the agent's value is decreasing in $k$ (by the envelope theorem, $\frac{d V}{d k}=\frac{\partial V}{\partial k}=\sum \alpha_{i} \frac{\partial q^{(i)}}{\partial k}$ ), and the resulting equilibrium preserves the properties in Proposition 3-except the property 1 when $\alpha$ is on the top region of $\mathcal{W}_{1}$. The reason that this property fails is rather mechanical. It is clear that by design, when $\alpha$ is on the top region of $\mathcal{W}_{1}$, a smaller $\alpha_{2}$ (keeping $\alpha_{1}$ constant) makes the selling position closer to the boundary $B^{k}$-and therefore a lower $p^{(1)}$. However, since $\mathcal{W}_{1}$ is small when $k$ is small, this exception is of little consequence.

\section{References}

Allen, F., and A. Santomero. 1997. The Theory of Financial Intermediation. Journal of Banking and Finance 21:1461-85.

Barry, C. B., C. Muscarella, J. Peavy, and M. Vetsuypens. 1990. The Role of Venture Capital in the Creation of Public Companies: Evidence from the Going Public Process. Journal of Financial Economics 27:447-72.

Bhattacharya, U., P. Reny, and M. Spiegel. 1995. Destructive Inference in an Imperfectly Competitive MultiSecurity Market. Journal of Economic Theory 65:136-70.

Caballe, J., and M. Krishnan. 1994. Imperfect Competition in a Multi-Security Market with Risk-Neutrality. Econometrica 62:695-704.

Chone, P., and J. C. Rochet. 1998. Ironing, Sweeping, and Multidimensional Screening. Econometrica 66:783826.

Dahiya, S., M. Puri, and A. Saunders. 2001. Bank Borrowers and Loan Sales: New Evidence on the Uniqueness of Bank Loans. Working Paper, Duke University.

Das, S., D. Duffie, N. Kapadia, and L. Saita. 2007. Common Failings: How Corporate Defaults Are Correlated. Journal of Finance 62:93-117.

Das, S., L. Freed, G. Geng, and N. Kapadia. 2006. Correlated Default Risk. Journal of Fixed Income 16:7-32.

DeMarzo, P. 2003. The Pooling and Tranching of Securities: A Model of Informed Intermediation. Working Paper, Stanford University.

DeMarzo, P. 2005. The Pooling and Tranching of Securities: A Model of Informed Intermediation. Review of Financial Studies 18:1-35.

Demsetz, R. 2000. Bank Loan Sales: A New Look at the Motivations for Secondary Market Activity. Journal of Financial Research 23:197-222. 
Diamond, D. 1984. Financial Intermediation and Delegated Monitoring. Review of Economic Studies 51:393-414.

Engers, M. 1987. Signalling with Many Signals. Econometrica 55:663-74.

Fudenberg, D., and J. Tirole. 1991. Game Theory. Cambridge, MA: The MIT Press.

Gorton, G., and G. Pennacchi. 1995. Banks and Loan Sales: Marketing Nonmarketable Assets. Journal of Monetary Economies 35:389-411.

Gande, A., and A. Saunders. 2005. Are Banks Still Special When There Is a Secondary Market for Loans? Working Paper, New York University-Stern.

Grinblatt, M., and C. Hwang. 1989. Signalling and the Pricing of New Issues. Journal of Finance 44:393-420.

He, Z. 2005. Cross-Signalling of Correlated Asset-Sales with Private Information. Working Paper, Northwestern University.

Hughes, P. 1986. Signalling by Direct Disclosure under Asymmetric Information. Journal of Accounting and Economics 8:119-42.

Ivashina, V. 2007. Agency Effects on Syndicated Loan Rates. Working Paper, Harvard Business School.

Kyle, A. 1985. Continuous Auctions and Insider Trading. Econometrica 53:1315-35.

Leland, H., and D. Pyle. 1977. Information Asymmetry, Financial Structure, and Financial Intermediation. Journal of Finance 32:371-87.

Mailath, G. 1987. Incentive Compatibility in Signalling Games with a Continuum of Types. Econometrica $55: 134-66$.

Mas-Colell, A., M. Whinston, and J. Green. 1995. Microeconomic Theory. New York: Oxford University Press.

Pavel, C., and D. Phillis. 1987. Why Commercial Banks Sell Loans: An Empirical Analysis. Federal Reserve Bank of Chicago Economic Perspectives 14:3-14.

Pennacchi, G. 1988. Loan Sales and the Cost of Bank Capital. Journal of Finance 43:375-96.

Riley, J. 1979. Informational Equilibrium. Econometrica 47:331-59.

Simons, K. 1993. Why Do Banks Syndicate Loans? New England Economic Review (January/February):45-52.

Sorenson, O., and T. E. Stuart. 2001. Syndication Networks and the Spatial Distribution of Venture Capital Investments. American Journal of Sociology 106:1546-88.

Sufi, A. 2007. Information Asymmetry and Financing Arrangements: Evidence from Syndicated Loans. Journal of Finance 62:629-68. 